\title{
An Efficient Algorithm for the 1D Total Visibility-Index Problem and Its Parallelization
}

\author{
PEYMAN AFSHANI, MADALGO, Aarhus University \\ MARK DE BERG, TU Eindhoven \\ HENRI CASANOVA and BEN KARSIN, University of Hawaii at Manoa \\ COLIN LAMBRECHTS, TU Eindhoven \\ NODARI SITCHINAVA, University of Hawaii at Manoa \\ CONSTANTINOS TSIROGIANNIS, MADALGO, Aarhus University
}

Let $T$ be a terrain and $P$ be a set of points on its surface. An important problem in Geographic Information Science (GIS) is computing the visibility index of a point $p$ on $P$, that is, the number of points in $P$ that are visible from $p$. The total visibility-index problem asks for the visibility index of every point in $P$.

We present the first subquadratic-time algorithm to solve the one-dimensional total-visibility-index problem. Our algorithm uses a geometric dualization technique to reduce the problem to a set of instances of the red-blue line segment intersection counting problem, allowing us to find the total visibility-index in $O\left(n \log ^{2} n\right)$ time. We implement a naive $O\left(n^{2}\right)$ approach and four variations of our algorithm: one that uses an existing red-blue line segment intersection counting algorithm and three new approaches that leverage features specific to our problem. Two of our implementations allow for parallel execution, requiring $O\left(\log ^{2} n\right)$ time and $O\left(n \log ^{2} n\right)$ work in the CREW PRAM model.

We present experimental results for both serial and parallel implementations on synthetic and real-world datasets using two hardware platforms. Results show that all variants of our algorithm outperform the naive approach by several orders of magnitude. Furthermore, we show that our special-case red-blue line segment intersection counting implementations out-perform the existing general-case solution by up to a factor 10 . Our fastest parallel implementation is able to process a terrain of more than 100 million vertices in under 3 minutes, achieving up to $85 \%$ parallel efficiency using 16 cores.

CCS Concepts: • Theory of computation $\rightarrow$ Computational geometry; Data structures design and analysis; Parallel algorithms; Divide and conquer;

Additional Key Words and Phrases: Terrain visibility, computational geometry, parallel algorithms, persistent data structures

\section{ACM Reference format:}

Peyman Afshani, Mark de Berg, Henri Casanova, Ben Karsin, Colin Lambrechts, Nodari Sitchinava, and Constantinos Tsirogiannis. 2018. An Efficient Algorithm for the 1D Total Visibility-Index Problem and Its Parallelization. F. Exp. Algorithmics 23, 2, Article 2.3 (July 2018), 23 pages.

https://doi.org/10.1145/3209685

Authors' addresses: P. Afshani and C. Tsirogiannis, Aarhus Universitet, Åbogade 34, 8200 Aarhus, Denmark; emails: \{peyman, constant\}@cs.au.dk; M. de Berg and C. Lambrechts, Department of Computer Science, TU Eindhoven, PO Box 513, 5600 MB Eindhoven, The Netherlands; emails: \{M.T.d.Berg, c.lambrechts\}@tue.nl; H. Casanova, B. Karsin, and N. Sitchinava, University of Hawaii, POST 317, 1680 East-West Road, Honolulu, HI 96822, USA; emails: \{henric, karsin, nodari\}@ hawaii.edu.

Permission to make digital or hard copies of all or part of this work for personal or classroom use is granted without fee provided that copies are not made or distributed for profit or commercial advantage and that copies bear this notice and the full citation on the first page. Copyrights for components of this work owned by others than ACM must be honored. Abstracting with credit is permitted. To copy otherwise, or republish, to post on servers or to redistribute to lists, requires prior specific permission and/or a fee. Request permissions from permissions@acm.org.

(C) 2018 ACM 1084-6654/2018/07-ART2.3 \$15.00

https://doi.org/10.1145/3209685 


\section{INTRODUCTION}

Analyzing terrains to determine locations with special properties is a common objective in Geographic Information Science (GIS). One such property is visibility. In particular, one often wants to find points on a terrain that are highly visible or, conversely, points that are hardly visible. Example applications include the placement of telecommunication towers, placement of fire guard towers, surveying archaeological sites, military logistics, or surveying building sites. Thus, in recent years there has been a fair amount of work in the GIS literature dedicated to visibility analysis and the computations it entails (see the survey by Floriani and Magillo [12]), with many proposed algorithms [10,11, 13, 17, 24, 26] as well as publicly available implementations [1,2].

To automate terrain analysis, real-world terrains are approximated by digital models, with one of the most popular models being the digital elevation model (DEM). A DEM is a grid of square cells, where each cell is assigned an elevation (which typically corresponds to the elevation of the point on the terrain that appears at the center of the cell).

Let terrain $T$ be a grid with $N=n^{2}$ total cells. Given two cells $c$ and $c^{\prime}$ with (x,y)-coordinates $\left(c_{x}, c_{y}\right)$ and $\left(c_{x}^{\prime}, c_{y}^{\prime}\right)$ and assuming that $c_{x}<c_{x}^{\prime}$, the cells are visible to each other if the line segment $\overline{c c^{\prime}}$ that connects their center-points does not cross on the $x y$-domain any other cell $g$ such that $\overline{c g}$ has slope greater than $\overline{c c^{\prime}}$ (that is, if $\left(g_{y}-c_{y}\right) /\left(g_{x}-c_{x}\right)>\left(c_{y}^{\prime}-c_{y}\right) /\left(c_{x}^{\prime}-c_{x}\right)$, where $\left(g_{x}, g_{y}\right)$ are the (x,y)-coordinates of cell $g$ ). We define the visibility index of cell $c \in T$ to be the number of cells in $T$ that are visible from $c$. The total visibility-index problem (also known as cumulative viewshed [27]) consists of finding the visibility index for every $c \in T$. One way to solve the total visibility-index problem on $T$ is to compute the viewshed of each cell $c$ of $T$, that is, to explicitly compute for each cell $c$ which other cells of $T$ are visible from $c$. With the algorithm of Van Kreveld [26] this takes $O(N \log N)$ time per cell, leading to a total running time of $O\left(N^{2} \log N\right)$. Even for moderately sized DEMs, this is infeasible in practice, let alone for modern DEM datasets, which can consist of hundreds of millions of cells. One solution is to use a heuristic that approximates the visibility $[11,24]$. Another is to observe that computing the viewsheds of different cells can be done independently and to solve a large number of single-viewshed computations in parallel $[5,9,20,21,28]$. Still, such approaches are not suitable for large DEMs. The fundamental problem is that one cannot afford to explicitly compute all visible cells for each cell $c$ of $T$, as this may produce an output of size $\Omega\left(N^{2}\right)$. Note that the total visibility index problem does not require us to explicitly compute the viewshed of each cell in $\mathrm{T}$; it only requires us to compute the number of cells that are visible from each cell, and therefore the output size for this problem is $\Theta(N)$.

So far, finding a subquadratic algorithm to solve the $1 \mathrm{D}$ total visibility-index problem remains an open problem. Surprisingly, no efficient algorithm has been proposed even for the one-dimensional (1D) version of the problem. In the $1 \mathrm{D}$ problem, the terrain $T$ is an $x$-monotone polyline with $n$ vertices. Similarly to the 2D problem, the goal in the one-dimensional version is to compute for each vertex $v$ in the polyline the number of vertices visible from $v$. We call this problem the $1 D$ total visibility-index problem. Note that on a $1 \mathrm{D}$ terrain $T$ with $n$ vertices, the visibility-index of a single vertex $v$ can be computed in $\Theta(n)$ time; this could be done by moving away from $v$ one vertex at a time and maintaining two rays that define the horizon to the left and right of $v$. Using this method to compute the visibility-index for each vertex independently, we can compute the total visibility-index of $T$ in $O\left(n^{2}\right)$ time. We refer to this simple algorithm as NAIVE. Despite its simplicity and disappointing quadratic performance, to the best of our knowledge, this is the best known solution for this problem to date.

The $1.5 \mathrm{D}$ terrain-guarding problem (TGP), which is highly related to the $1 \mathrm{D}$ total visibility-index problem, has been extensively studied [14, 15, 18, 22]. The terrain-guarding problem involves finding the minimum number of points needed to view an entire one-dimensional set of vertices. While TGP is related to the total visibility-index and involves visibility, solving it is known to be 
NP-hard. Thus, previous results that solve TGP provide approximate solutions [14, 15, 22]. Hurtado et al. [18] present a solution to a variant of TGP by considering the viewshed (list of visible points) of $m$ viewpoints (or guards). By sweeping a one-dimensional terrain (an x-monotone polyline of vertices) and maintaining a list of vertices that are dominated, they find the viewshed of $m$ points in $O(n+m \log m)$ time. While Hurtado et al. [18] find the list of points visible from $m$ viewpoints, we are concerned with finding the number of points on the terrain visible for each point. Therefore, it is not clear how their techniques can be used to solve the total visibility-index problem efficiently.

Our Contributions. In this article, we present an algorithm that solves the 1D total visibility-index problem for a terrain of $n$ vertices in $O\left(n \log ^{2} n\right)$ time. Our algorithm uses a geometric dualization technique, which transforms the visibility problem into a set of instances of the $2 D$ red-blue line segment intersection-counting problem. In fact, we show that the instances of red-blue line segments that we have to process have characteristics that allow us to develop a simpler algorithm for counting intersections. This new intersection counting algorithm performs faster in practice than existing algorithms that solve the general red-blue line segment intersecting problem [23]. We also show how to parallelize our algorithm while keeping the overall work (total operations) the same. In particular, we present an adaptation of our algorithm in the CREW PRAM model [19], which requires $O\left(\log ^{2} n\right)$ time and $O\left(n \log ^{2} n\right)$ work. We implement the NAIve $O\left(n^{2}\right)$ algorithm, as well as four variations of our algorithm: REDBLUE employs an existing red-blue segment intersection counting algorithm [23], while SWEEP, PARAoT, and LiNPAR implement three versions of our new intersection counting technique. Both PARAoT and LINPAR allow for parallel execution to improve performance. LINPAR employs a space-efficient data structure to reduce the $O(n \log n)$ memory required by the simpler PARAoT.

We evaluate the performance of our implementations on large synthetic datasets, as well as datasets generated from real-world terrain maps, showing that all four implementations of our algorithm outperform the naive solution by several orders of magnitude. Additionally, we show that implementations employing our new intersection counting algorithm are able to reduce execution time by up to $18.69 \times$ over the existing general-case solution on our two hardware platforms (detailed in Section 5.1). We provide a detailed analysis of the performance of our two parallel implementations on two hardware platforms. Results indicate that our space-efficient solution, LiNPAR, provides the highest peak performance and is capable of processing over 100 million vertices in under 3 minutes, achieving up to $85 \%$ parallel efficiency.

\section{PRELIMINARIES}

Let $T[1 . . n]$ be a one-dimensional terrain defined by an array of elevation values in $\mathbb{R}^{1}$. Element $T[i]$ stores the elevation $h_{i}$ of the $i$ th cell of the terrain. The array $T$ defines an $x$-monotone polyline obtained by connecting the vertices $p_{i}:=\left(i, h_{i}\right)$ for $i=1, \ldots, n$ in order. Let $P=\left(p_{1}, p_{2}, \ldots, p_{n}\right)$ denote the sequence of these vertices ordered by their $x$-coordinates, and let $P[l: r]$ denote the subset of vertices $\left(p_{l}, \ldots, p_{r}\right)$. We say that a vertex $p_{j}$ is visible from $p_{i}\left(p_{i}\right.$ sees $\left.p_{j}\right)$, if all vertices $p_{k}$ between $p_{i}$ and $p_{j}$ lie strictly below the segment $\overline{p_{i} p_{j}}$. Based on this definition, we conclude that a vertex is visible from itself, and if vertex $p_{j}$ is visible from vertex $p_{i}$, then $p_{i}$ is also visible from $p_{j}$. We define the visibility ray from $p_{i}$ to $p_{j}$, denoted $\overrightarrow{p_{i} p_{j}}$, as the ray that starts at $p_{i}$ and passes through $p_{j}$. We define the visibility ray $\overrightarrow{p_{i} p_{i}}$ as the vertical ray that crosses $p_{i}$ and points downwards. Let $v_{\text {vert }}\left(p_{i}\right)$ denote the ray that starts at $p_{i}$ and points vertically up. We define the angle of the visibility ray $\overrightarrow{p_{i} p_{j}}$ as the smallest angle between $\overrightarrow{p_{i} p_{j}}$ and $v_{\text {vert }}\left(p_{i}\right)$. We use $\alpha\left(\overrightarrow{p_{i} p_{j}}\right)$ to denote this angle.

One of the key concepts that we use in our analysis is that of the critical ray. Let $l, i$, and $r$ be three positive integers such that $l \leq i \leq r \leq n$. The left critical ray of point $p_{i}$ with respect 


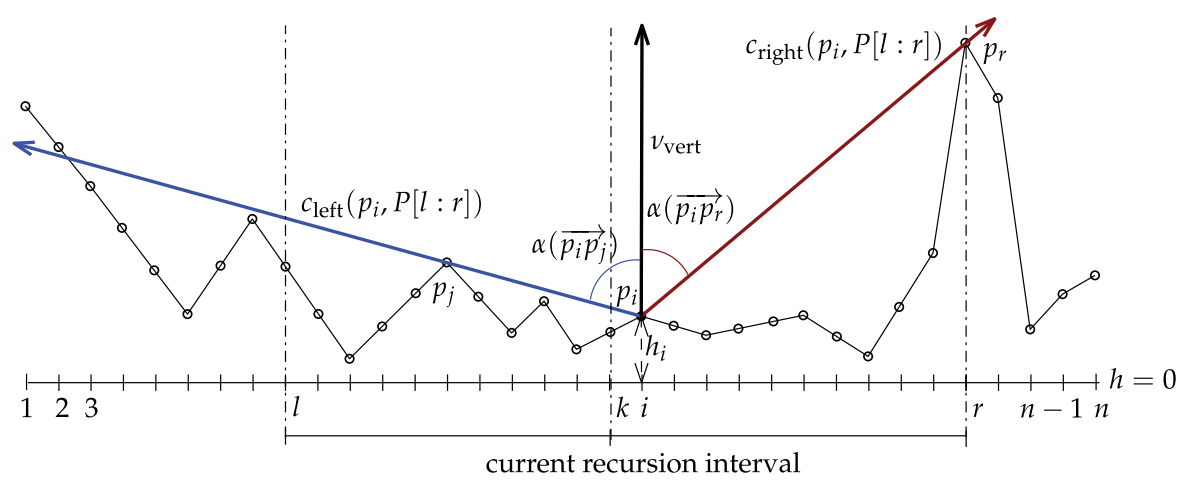

Fig. 1. Illustration of an one-dimensional terrain, together with the critical rays from vertex $p_{i}$.

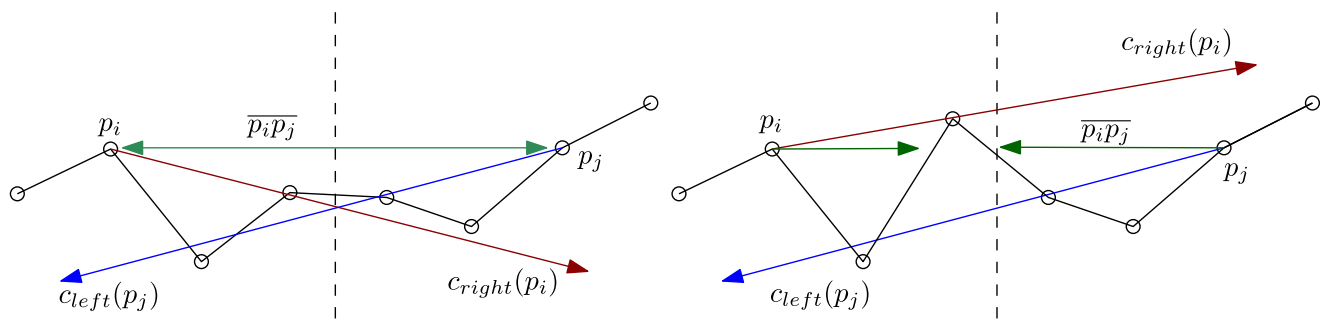

Fig. 2. Illustration of the intuition behind Lemma 2.1. Left: Example where both points are above critical rays. Right: Example where a point is below a critical ray.

to $P[l: r]$, is the visibility ray $\overrightarrow{p_{i} p_{s}}$ with the smallest $\alpha\left(\overrightarrow{p_{i} p_{s}}\right)$ among all rays $\overrightarrow{p_{i} p_{k}}$ with $l \leq k \leq i$. We denote this ray by $c_{\text {left }}\left(p_{i}, P[l: r]\right)$. If $i=l$, then $c_{\text {left }}\left(p_{i}, P[l: r]\right)$ is defined as the ray pointing vertically down from $p_{i}$. The right critical ray, denoted $c_{\text {right }}\left(p_{i}, P[l: r]\right)$, of $p_{i}$ is the visibility ray $\overrightarrow{p_{i} p_{t}}(i \leq t \leq r)$ with the smallest $\alpha\left(\overrightarrow{p_{i} p_{t}}\right)$ (or pointing vertically down from $p_{i}$ if $\left.i=r\right)$. See Figure 1 for an illustration of these rays. We can use critical rays to determine visibility between two points, as the following lemma shows.

LeMma 2.1. Two points $p_{i} \in P[l: k]$ and $p_{j} \in P[k+1: r]$ are visible from each other if and only if $p_{i}$ is above $c_{\text {left }}\left(p_{j}, P[k+1: r]\right)$ and $p_{j}$ is above $c_{\text {right }}\left(p_{i}, P[l: k]\right)$.

Proof. Let $c_{\text {right }}:=c_{\text {right }}\left(p_{i}, P[l: k]\right)$ be the right critical ray of $p_{i}$ and let $c_{\text {left }}:=c_{\text {left }}\left(p_{j}\right.$, $P[k+1: r])$ be the left critical ray of $p_{j}$. Consider the line segment $\overline{p_{i} p_{j}}$. Assume that $p_{i}$ is above $c_{\text {left }}$ and that $p_{j}$ is above $c_{\text {right }}$. Then all points $P[i: k]$ are below $\overline{p_{i} p_{j}}$, since by definition they lie below or on $c_{\text {right }}$.

Symmetrically, all points $P[k+1: j]$ are below $\overline{p_{i} p_{j}}$, due to $c_{\text {left }}$. Hence, $p_{i}$ and $p_{j}$ are visible from each other. Now assume that $p_{i}$ and $p_{j}$ are visible from each other. That means that all points $P[i+1: j-1]$ are below $\overline{p_{i} p_{j}}$. All points that can possibly determine $c_{\text {right }}$ and $c_{\text {left }}$ are therefore also below $\overline{p_{i} p_{j}}$. Hence $p_{i}$ is above $c_{\text {left }}$ and $p_{j}$ is above $c_{\text {right }}$.

Figure 2 illustrates the intuition behind the previous lemma. Note that, while we use the restriction that visibility requires points to be above critical rays, this is just a matter of definition. Changing our visibility definition to include equality would not change the overall algorithm design or performance. 


\section{DESCRIPTION OF THE ALGORITHM}

Let $T$ be a one-dimensional terrain and let $P$ be the set of its vertices. To compute the total visibilityindex on $T$, we consider the following divide-and-conquer approach: First, we split the input polyline $P$ into two subsets of equal size, and we recursively continue this process. After computing the total visibility-index for each trivial base case, we move up in the hierarchy of recursive calls. At each step, we combine the results that we computed for two consecutive subsets $P[l: k]$ and $P[k+1: r]$ to produce the total visibility-index for subset $P[l: r]$. For each subset that we process, together with computing the visibility-index for each vertex $p$ in the subset, we also construct the left and right critical ray of $p$ with respect to this subset. At any point during this recursive execution, we use an array VisIndex such that VisIndex [i] stores the total visibility-index of $p_{i}$ computed in all previous levels of recursion. Suppose that, at some point during this recursion, we have already calculated the total visibility-index for two subsets $P[l: k]$ and $P[k+1: r]$, and we need to produce the result for their union $P[l: r]$. To do this, we need to compute for each $p_{i} \in P[l: k]$ the number of vertices of $P[k+1: r]$ that are visible to $p_{i}$ and add this number to VisIndex [i]; similarly, for each $p_{j} \in P[k+1: r]$ we need to compute the number of points of $P[l: k]$ that are visible from $p_{j}$ and add this to VisIndex[j]. We define Bipartite Visibility as this problem of finding the number of visible vertices only between elements of two distinct subsets. To reduce each recursive step of our divide-and-conquer algorithm to an instance of Bipartite Visibility, we define the following invariants that must be satisfied for each $p_{i} \in P[l: k]$, respectively, $p_{j} \in P[k+1: r]$ :

- VisIndex $[i]$ and VisIndex $[j]$ have been computed within $P[l: k]$ and $P[k+1, r]$, respectively,

- $c_{\text {right }}\left(p_{i}, P[l: k]\right)$ and $c_{\text {left }}\left(p_{i}, P[l: k]\right)$ correspond to the maximum and minimum slope rays, respectively, between $p_{i}$ and any $p_{l} \in P[l: k]$,

- symmetrically, $c_{\text {right }}\left(p_{j}, P[k+1: r]\right)$ and $c_{\text {left }}\left(p_{j}, P[k+1: r]\right)$ correspond to the maximum and minimum slope rays, respectively, between $p_{j}$ and any $p_{m} \in P[k+1: r]$.

- the upper convex hulls of the vertices of $P[l: k]$ and $P[k+1: r]$ have been computed from the previous recursive step. These are needed to update the critical rays for the next recursive step (this process is detailed in Section 3.3).

With the above invariants satisfied, we solve an instance of Bipartite Visibility to compute the number of visible vertices between the two distinct subsets $P[l: k]$ and $P[k+1: r]$. We denote the entire divide-and-conquer algorithm that computes the total visibility-index of $P$ as 1DVisibilityIndex. The runtime of 1DVisibilityIndex on $P$ is given by the recurrence $\tau(n)=$ $2 \tau(n / 2)+f(n)$, where $f(n)$ is the time it takes to solve Bipartite Visibility for $P[1: n / 2]$ and $P[n / 2+1: n]$. Therefore, the algorithmic performance of this divide-and-conquer approach depends on an efficient solution for Bipartite Visibility. This section focuses on describing an algorithm that solves Bipartite Visibility in $O(n \log n)$ time and $O(n)$ space by reducing it to red-blue line segment intersection counting and using the work of Palazzi and Snoeyink [23] to solve it, leading to:

THEOREM 3.1. Let $T$ be an $1 D$ terrain that consists ofn vertices. We can compute the total visibilityindex of $T$ in $O\left(n \log ^{2} n\right)$ time, using $O(n)$ space.

Let $P[l: k]$ and $P[k+1: r]$ be two parts of the terrain for which we want to solve Bipartite Visibility. Recall that for all vertices in $P[l: k]$ we have already computed the right critical rays with respect to $P[l: k]$, and for all vertices in $P[k+1: r]$ we have computed the left critical rays with respect to $P[k+1: r]$. Let $p_{i}$ be a vertex in $P[l: k]$, and let $p_{j}$ be a vertex in $P[k+1: r]$. Recall that, according to Lemma 2.1, vertices $p_{i}$ and $p_{j}$ are visible to each other if both $p_{j}$ lies above the right critical ray of $p_{i}$, and $p_{i}$ lies above the left critical ray of $p_{j}$. Therefore, to compute the number of vertices in $P[k+1: r]$ that are visible from $p_{i}$, we could explicitly check if this condition holds 
for each $p_{j}$ in $P[k+1: r]$. This method, however, is inefficient as it requires that we check all possible pairs of vertices $p_{i}$, $p_{j}$ s.t. $p_{i} \in P[l: k]$ and $p_{j} \in P[k+1: r]$.

We improve on this naive solution by using geometric duality [7]. Instead of handling the actual critical rays of the input points, we dualize these rays: we construct exactly one dual half-line for the right critical ray of each vertex in $P[l: k]$, and one dual half-line for the left critical ray of each vertex in $P[k+1: r]$. We refer to the duals of the right critical rays as the red half-lines and denote them by $\rho$, and the duals of the left critical rays as the blue half-lines and denote them by $\beta$. We detail the construction of these dual half-lines in Section 3.1. As we will show with Lemma 3.2, we can construct the dual half-lines in such a way that the following property holds; a vertex $p_{i}$ in $P[l: k]$ and a vertex $p_{j}$ in $P[k+1: r]$ are visible if and only if the duals of their critical rays intersect. Hence, to compute the number of vertices in $P[k+1: r]$ that are visible from $p_{i}$, it suffices to count the number of blue half-lines that intersect with the dual of $c_{\text {right }}\left(p_{i}, P[l: r]\right.$ ) (which is a red half-line). Thus, to solve this instance of Bipartite Visibility, we need to count, for each red and each blue dual half-line, the number of intersections that it induces with half-lines of the opposite color. In Section 3.1 we describe how we can do this efficiently in $O(n \log n)$ time.

In addition to computing Bipartite Visibility, at each recursive step of 1DVisibilityIndex, the critical rays of each vertex must be updated with respect to the new subset that will contain it (e.g., $P[l: r])$. Therefore, after computing Bipartite Visibility between $P[l: k]$ and $P[k+1: r]$, we must update the critical rays of each vertex with respect to $P[l: k] \cup P[k+1: r]$. We detail the process of updating critical rays in Section 3.3. The remainder of the current section details the steps of 1DVisibilityInDEX, with the pseudocode of the overall algorithm presented in Algorithm 1.

The approach that we describe for Bipartite Visibility is similar to the method used by Ben-Moshe et al. [4] for computing the visibility graph between a set of points inside a polygon. However, since their goal is to construct the actual visibility graph (which can have quadratic

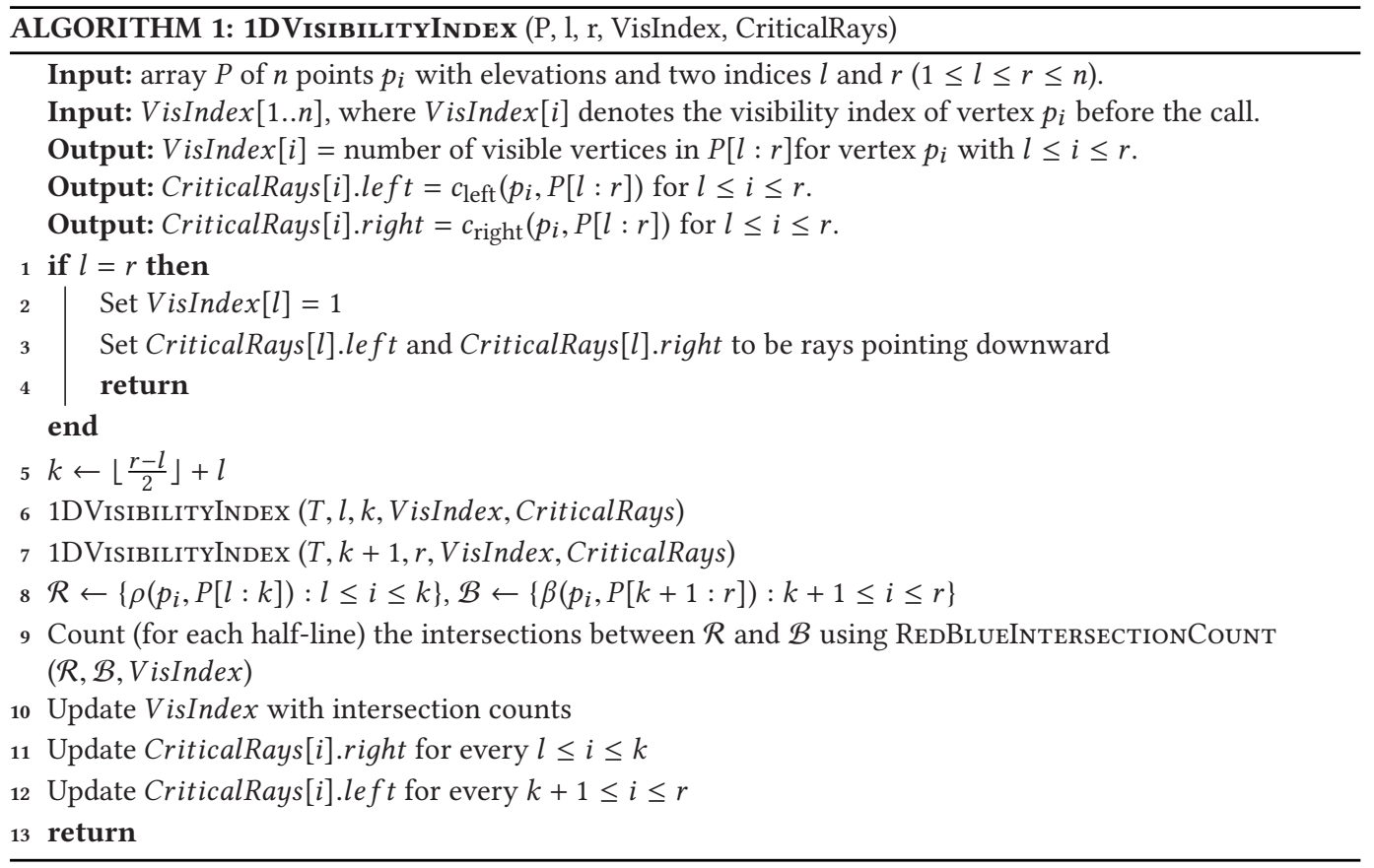



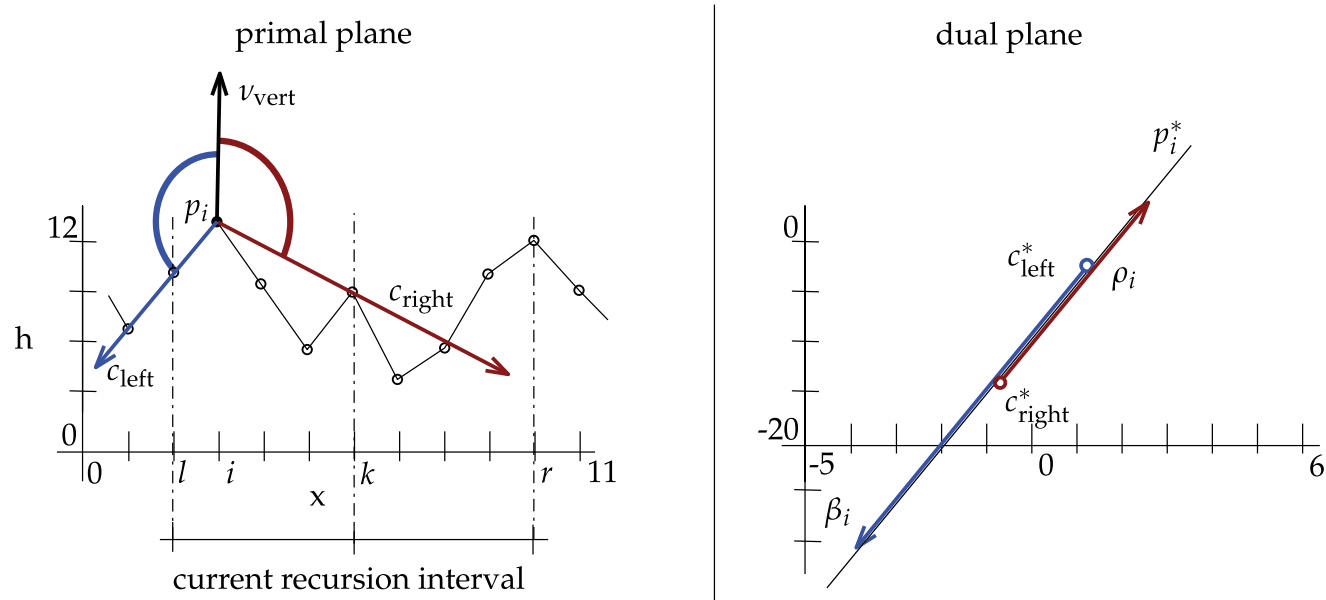

Fig. 3. An example of a terrain, its critical rays and their corresponding dual half-lines.

size with respect to the input), they use an output-sensitive approach that is much slower than the methods that we describe for counting red-blue line segment intersections.

\subsection{Constructing Dual Rays and Counting Red-Blue Intersections}

In this section we describe how we utilize duality to reduce the Bipartite Visibility problem to the red-blue line segment intersection counting problem. We can thereby solve it using existing methods.

The dual of a point $p_{i}:\left(i, h_{i}\right)$ is defined as the line $p_{i}^{*}: y=i x-h_{i}$, and the dual of a line $l: y=$ $a x+b$ as the point $l^{*}:(a,-b)$. Let $P[l: k]$ be a subset of consecutive vertices in the input terrain. Consider vertex $p_{i} \in P[l: k]$ with the critical rays $c_{\text {right }}\left(p_{i}, P[l: k]\right)$ and $c_{\text {left }}\left(p_{i}, P[l: k]\right)$ lying along the lines $y=a_{r} x+b_{r}$ and $y=a_{l} x+b_{l}$, respectively. Let $\rho\left(p_{i}, P[l: k]\right)$ be the dual of the set of lines that pass through $p_{i}$ and have slopes strictly larger than $a_{r}$. Since each line passes through the point $p_{i}$, in the dual space each line becomes a point that falls on the line corresponding to the dual of $p_{i}$. Thus, $\rho\left(p_{i}, P[l: k]\right)$ is an open half-line with endpoint $c_{\text {right }}^{*}=\left(a_{r},-b_{r}\right)$, extending to $+\infty$, and supported by the line $y=i x-h_{i}$. Similarly, let $\beta\left(p_{i}, P[l: k]\right)$ be the dual of the set of lines that pass through $p_{i}$ and with slopes strictly smaller than $a_{l}$. Thus, $\beta\left(p_{i}, P[l: k]\right)$ is an open half-line that is collinear with $\rho\left(p_{i}, P[l: k]\right)$ and and extends from $c_{\text {left }}^{*}=\left(a_{l},-b_{l}\right)$ to $-\infty$. Note that $\rho\left(p_{i}, P[l: k]\right)$ and $\beta\left(p_{i}, P[l: k]\right)$ are open half-lines and they therefore do not contain their endpoints $c_{\text {right }}^{*}$ and $c_{\text {left }}^{*}$, respectively. For simplicity, we refer to open half-lines simply as half-lines for the remainder of this article. Refer to Figure 3 for an example.

Lemma 3.2. Consider two points $p_{i} \in P[l: k]$ and $p_{j} \in P[k+1: r]$ and the critical rays $c_{\text {right }}\left(p_{i}, P[l: k]\right)$ and $c_{\text {left }}\left(p_{j}, P[k+1: r]\right)$, then $p_{i}$ and $p_{j}$ are visible from each other if and only if there is an intersection between dual half-lines $\rho\left(p_{i}, P[l: k]\right)$ and $\beta\left(p_{j}, P[k+1: r]\right)$.

Proof. Suppose $p_{i}$ and $p_{j}$ are visible from each other. Consider the line $l$ that passes through $p_{i}$ and $p_{j}$. The dual of $l$ is a point $l^{*}$. By Lemma $2.1, p_{i}$ must be above $c_{\text {left }}\left(p_{j}, P[k+1: r]\right)$. Therefore, the slope of $l$ must be smaller than the slope of $c_{\text {left }}\left(p_{j}, P[k+1: r]\right)$ and, consequently, $l^{*} \in \beta\left(p_{j}, P[k+1: r]\right)$. Similarly, by Lemma $2.1, p_{j}$ must be above $c_{\text {right }}\left(p_{i}, P[l: k]\right)$. Therefore, the slope of $l$ must be larger than the slope of $c_{\text {right }}\left(p_{i}, P[l: k]\right)$ and, consequently, $l^{*} \in \rho\left(p_{i}, P[l: k]\right)$. Since dual point $l^{*}$ belongs to both dual half-lines, they must be intersecting at $l^{*}$. 
Suppose $\beta\left(p_{j}, P[k+1: r]\right)$ and $\rho\left(p_{i}, P[l: k]\right)$ intersect at the dual point $q^{*}$. The dual point $q^{*}$ corresponds to a line $q$ that goes through both $p_{i}$ and $p_{j}$. Since $q^{*} \in \rho\left(p_{i}, P[l: k]\right)$, the slope of $q$ must be larger than the slope of $c_{\text {right }}\left(p_{i}, P[l: k]\right)$, i.e. $p_{j}$ must be above $c_{\text {right }}\left(p_{i}, P[l: k]\right)$. Similarly, since $q^{*} \in \beta\left(p_{j}, P[k+1: r]\right)$, the slope of $q$ must be smaller than the slope of $c_{\text {left }}\left(p_{j}, P[k+1: r]\right)$, i.e., $p_{i}$ must be above $c_{\text {left }}\left(p_{j}, P[k+1: r]\right)$. Therefore, by Lemma 2.1, $p_{i}$ and $p_{j}$ are visible from each other.

Lemma 3.2 allows us to solve the Bipartite Visibility problem by computing for each dual halfline $\beta\left(p_{j}, P[k+1: r]\right)$, how many half-lines $\rho\left(p_{i}, P[l: k]\right)$ it intersects, and vice versa. The next lemma is important for finding an efficient intersection counting algorithm.

Lemma 3.3. Let $p_{i}$ and $p_{j}, i \neq j$, be two points in $P[l: k]$. Then the dual half-lines $\rho\left(p_{i}, P[l: k]\right)$ and $\rho\left(p_{j}, P[l: k]\right)$ do not intersect. Similarly, $\beta\left(p_{i}, P[l: k]\right)$ and $\beta\left(p_{j}, P[l: k]\right)$ do not intersect.

Proof. Suppose for the sake of contradiction that $\rho\left(p_{i}, P[l: k]\right)$ and $\rho\left(p_{j}, P[l: k]\right)$ do intersect, which means that there is a visibility line $\overline{p_{i} p_{j}}$ between the $p_{i}$ and $p_{j}$ (in the primal plane). It also means that both $c_{\text {right }}\left(p_{i}, P[l: k]\right)$ and $c_{\text {right }}\left(p_{j}, P[l: k]\right)$ fall below $\overline{p_{i} p_{j}}$ (i.e., $\alpha\left(\overrightarrow{p_{i} p_{j}}\right)<$ $\alpha\left(c_{\text {right }}\left(p_{i}, P[l: k]\right)\right)$ and $\left.\alpha\left({\overrightarrow{p_{i} p_{j}}}\right)<\alpha\left(c_{\text {right }}\left(p_{j}, P[l: k]\right)\right)\right)$. By the definition of the critical ray, no visibility ray between two points in $P[l: k]$ can have a smaller angle $\alpha$ than the critical ray. Hence the angle must be equal to that of the critical ray and therefore the visibility line is the critical ray. This means that the intersection is at the starting point of the dual half-line. Since $\rho\left(p_{i}, P[l: k]\right)$ and $\rho j l k$ are open half-lines, the starting points are not part of them and, therefore, they do not intersect. The proof for $\beta\left(p_{i}, P[l: k]\right)$ and $\beta\left(p_{j}, P[l: k]\right)$ is symmetric.

Palazzi and Snoeyink [23] present an algorithm that computes, in $O(n \log n)$ time, the total number of intersections between a set of non-self-intersecting (red) line segments and another set of non-self-intersecting (blue) segments. Half-lines are a special case of line segments, where one endpoint is at $\infty$ (or $-\infty)$. Thus, to use the general intersection counting algorithm of Palazzi and Snoeyink [23], we replace the unbounded side of each half-line with an endpoint with very large $\mathrm{x}$-coordinate, which is sufficient to capture any intersections that occur with our datasets. We note that the algorithm by Palazzi and Snoeyink produces only the total number of red-blue intersections (i.e., a single number), while we require intersection counts for each half-line. However, we can modify their algorithm to produce the desired result without impacting asymptotic performance by simply keeping a tally of intersections for each segment during the algorithm. Any further differences between our problem and general red-blue segment intersection counting only serve to restrict the the problem, which we use to develop a more efficient solution in the following section.

\subsection{A Practical Algorithm for Red-Blue Intersection Counting}

While using the (modified) red-blue segment intersection algorithm of Palazzi and Snoeyink [23] provides an $O(n \log n)$ solution to Bipartite Visibility, it works for any red-blue line segments. As a result, it is more complex than it has to be for our problem. Instead, in this subsection we present a simple plane sweep algorithm to count the intersections between duals of right and left critical rays. This plane sweep algorithm exploits some features of the dual half-lines of critical rays.

Let $\mathcal{R}=\left\{\rho\left(p_{i}, P[l: k]\right)\right\}$ and $\mathcal{B}=\left\{\beta\left(p_{j}, P[k+1: r]\right)\right\}$ be the set of red and blue self-nonintersecting open half-lines (i.e., no half-lines intersect others of the same color). To describe our algorithm, we need to introduce additional notation. We denote the $x$ - and $y$-coordinate of a vertex $p$ by $p_{x}$ and $p_{y}$, respectively. Given any half-line $\lambda$, we denote its endpoint by $\lambda_{x, y}$ and the $x$ - and $y$-coordinates of the endpoint by $\lambda_{x}$ and $\lambda_{y}$, respectively, i.e., $\lambda_{x, y}=\left(\lambda_{x}, \lambda_{y}\right)$. The $y$-coordinate of $\lambda$ evaluated at $x$ is denoted by $\lambda(x)$. That is, if $\lambda$ is defined at $x$ then vertex $p_{x, \lambda(x)}=(x, \lambda(x)) \in \lambda$. 


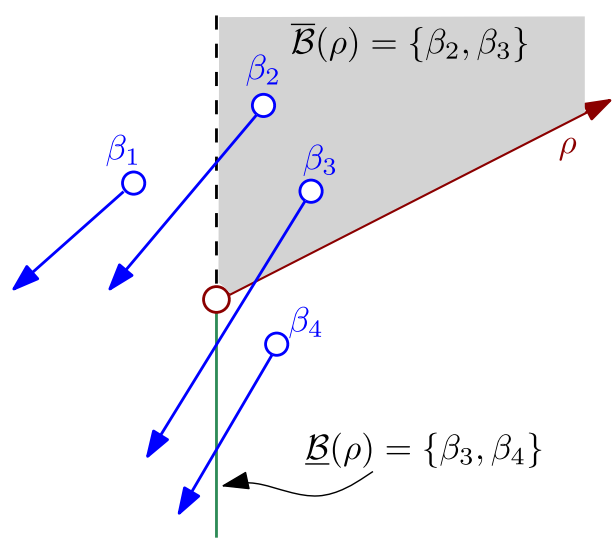

Fig. 4. An illustration of $\underline{\mathcal{B}}(\rho)$ and $\overline{\mathcal{B}}(\rho)$.

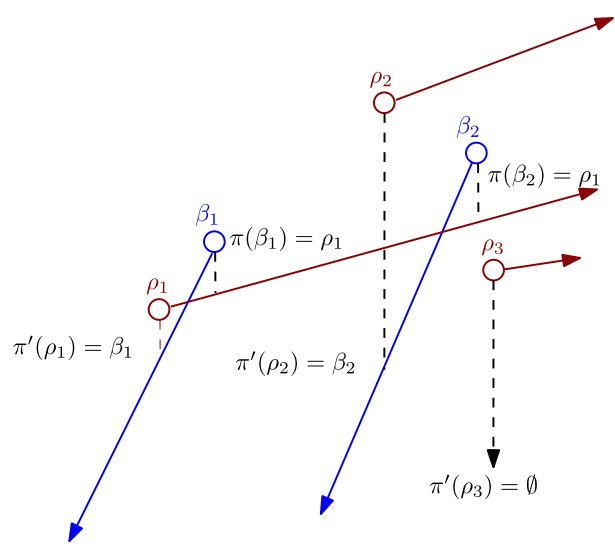

Fig. 5. Illustration of $\pi^{\prime}(\rho)$ and $\pi(\beta)$ for several half-lines.

If $\lambda$ is not defined at $x$, then we say $\lambda(x)$ is undefined. Finally, we say a vertex $q$ is above (respectively, below) a half-line $\lambda$, if $\lambda\left(q_{x}\right)$ is defined and $q_{y}>\lambda\left(q_{x}\right)$ (respectively, $q_{y}<\lambda\left(q_{x}\right)$ ). If $\lambda\left(q_{x}\right)$ is undefined, then the above-below relationship between $q$ and $\lambda$ is undefined.

The following lemma is the key for developing a simple plane sweep algorithm for our red-blue half-line intersection counting problem.

Lemma 3.4. Any two half-lines $\rho \in \mathcal{R}$ and $\beta \in \mathcal{B}$ intersect if and only if the endpoint $\rho_{x, y}$ is above $\beta$ and the endpoint $\beta_{x, y}$ is above $\rho$.

Proof. Suppose $\rho_{x, y}$ is above $\beta$ and $\beta_{x, y}$ is above $\rho$. There must be a point $q$ with $\rho_{x}<q_{x}<\beta_{x}$ s.t. $\rho\left(q_{x}\right)=\beta\left(q_{x}\right)$. Since $\rho$ is continuous for all $x>\rho_{x}$ and $\beta$ is continuous for all $x<\beta_{x}, \rho$ and $\beta$ intersect at $q_{x}$. This can be seen in the example in Figure 5 .

In the primal space, all points from the left merge set have smaller $\mathrm{x}$-coordinates than any point from the right set. Therefore, all $\rho \in \mathcal{R}$ have a smaller slope than all $\beta \in \mathcal{B}$. It follows that, if $\rho$ and $\beta$ intersect at $q$, then $\rho(a)>\beta(a)$ and $\beta(b)>\rho(b)$ for all $a<q_{x}<b$. Since $\rho_{x}$ has the smallest $\mathrm{x}$-coordinate for which $\rho$ is defined, then $\rho_{x}<q_{x}$. Therefore, $\rho_{x, y}$ is above $\beta$. Conversely, $\beta_{x}$ is the largest x-coordinate of $\beta$, so $\beta_{x}>q_{x}$. Thus, $\beta_{x, y}$ is also above $\rho$.

To compute the number of blue half-lines in $\mathcal{B}$ that each $\rho \in \mathcal{R}$ intersects, consider the following subsets of blue half-lines (see Figure 4):

- $\overline{\mathcal{B}}(\rho)$ : blue half-lines $\beta \in \mathcal{B}$ with endpoints that are above $\rho$ (i.e., $\beta_{y}>\rho\left(\beta_{x}\right)$ )

- $\underline{B}(\rho)$ : blue half-lines $\beta \in \mathcal{B}$ that are below $\rho_{x, y}$ (i.e., $\beta\left(\rho_{x}\right)<\rho_{y}$ )

By Lemma 3.4, the set of blue half-lines that intersect $\rho$ is $\overline{\mathcal{B}}(\rho) \cap \mathcal{B}(\rho)$ and by the inclusionexclusion principle, its cardinality is $|\overline{\mathcal{B}}(\rho)|+|\underline{\mathcal{B}}(\rho)|-|\overline{\mathcal{B}}(\rho) \cup \underline{\mathcal{B}}(\rho)|$. Note that $\overline{\mathcal{B}}(\rho) \cup \underline{\mathcal{B}}(\rho)$ is the set of all blue half-lines with $x$-ranges that overlap with $\rho$, i.e., $\overline{\bar{B}}(\rho) \cup \underline{\mathcal{B}}(\rho)=\left\{\beta \in \mathcal{B}: \beta_{x}>\rho_{x}\right\}$. Figure 4 shows an example for a single red half-line and four blue half-lines.

Similarly, we define $\underline{\mathcal{R}}(\beta)$ and $\overline{\mathcal{R}}(\beta)$ and the number of red half-lines that intersect $\beta$ is equal to $|\overline{\mathcal{R}}(\beta)|+|\underline{\mathcal{R}}(\beta)|-|\overline{\mathcal{R}}(\beta) \cup \underline{\mathcal{R}}(\beta)|$. Thus, it remains to compute each of these quantities.

3.2.1 Computing $|\underline{\mathcal{B}}(\rho)|,|\underline{\mathcal{R}}(\beta)|,|\overline{\mathcal{B}}(\rho) \cup \underline{\mathcal{B}}(\rho)|$, and $|\overline{\mathcal{R}}(\beta) \cup \underline{\mathcal{R}}(\beta)|$. To compute $|\underline{\mathcal{B}}(\rho)|$, we sweep the dual plane from right to left with a sweep line $\ell$ that is perpendicular to the $x$-axis. 
During the sweep, we maintain a balanced binary search tree (BST) $\mathcal{T}$ that stores all blue halflines $\beta$ that intersect $\ell$, ordered by their slopes. Since blue half-lines do not intersect each other and continue to $-\infty$, this order remains consistent throughout the entire sweep. Thus, every time the sweep line encounters a blue half-line endpoint $\beta_{x, y}$, we insert $\beta$ to $\mathcal{T}$. Whenever the sweep line encounters the endpoint $\rho_{x, y}$ of a red half-line, the number of blue half-lines below $\rho$ is equal to the number of blue half-lines $\beta$ with $y$-coordinate $\beta\left(\rho_{x}\right)$ smaller than $\rho_{y}$. And since all blue half-lines in $\mathcal{T}$ are defined at the time of the sweep, the above-below relationship between the endpoint $\rho_{x, y}$ and all blue half-lines in $\mathcal{T}$ is well defined. Thus, we can compute $|\underline{\mathcal{B}}(\rho)|$ by performing a search in $\mathcal{T}$, comparing $\rho_{y}$ to $\beta\left(\rho_{x}\right)$. The result of this search is the rank of $\rho_{y}$ in the set of blue half-lines in $\mathcal{T}$, where $\operatorname{rank}(x)$ is defined as the number of elements in a sorted list with key value less than $x$. The rank of $\rho_{y}$ in the set of blue half-lines in $\mathcal{T}$ gives us $|\underline{B}(\rho)|-$ the number of blue half-lines below $\rho$.

To implement this plane sweep, we start by sorting $\mathcal{B}$ and $\mathcal{R}$ by the $x$-coordinates of their endpoints. Each insertion of a blue half-line in $\mathcal{T}$ takes $O(\log n)$ time. We can compute the rank of $\rho_{y}$ in $\mathcal{T}$ in $O(\log n)$ time by augmenting each node $v$ of $\mathcal{T}$ with the size of the subtree rooted at $v$. Thus, the total computation of $|\underline{B}(\rho)|$ for all $\rho \in \mathcal{R}$ takes $O(n \log n)$ time.

Note that the size of $\mathcal{T}$ when the sweep line encounters $\rho_{x, y}$ is $|\overline{\mathcal{B}}(\rho) \cup \underline{\mathcal{B}}(\rho)|$-the number of blue half-lines whose $x$-ranges overlap with $\rho$. During the computation we also record for each red half-line $\rho$ the blue half-line $\pi^{\prime}(\rho)$ that is immediately below $\rho$ (the predecessor of $\rho_{y}$ in the $\mathcal{T})$. Refer to Figure 5 for an illustration.

Computation of $|\underline{\mathcal{R}}(\beta)|$ is symmetric, with the sweep being performed from left to right. During the computation, we also record $|\overline{\mathcal{R}}(\beta) \cup \underline{\mathcal{R}}(\beta)|$ and $\pi(\beta)$-the red half-line that is immediately below the endpoint of $\beta$. The concepts of $\pi(\beta)$ and $\pi^{\prime}(\rho)$ will be used for computing $|\overline{\mathcal{B}}(\rho)|$ and $|\overline{\mathcal{R}}(\beta)|$, respectively.

3.2.2 Computing $|\overline{\mathcal{B}}(\rho)|$ and $|\overline{\mathcal{R}}(\beta)|$. The following description focuses on the computation of values $|\overline{\mathcal{B}}(\rho)|$; the computation of $|\overline{\mathcal{R}}(\beta)|$ is symmetric. Since computing $|\underline{\mathcal{B}}(\rho)|$ and $|\underline{\mathcal{R}}(\beta)|$ entails counting half-lines below each given endpoint, the above-below relationship is well defined at the time the sweep line hits the endpoint in question. Here, instead, we are counting the number of points above a half-line, which must be counted for every half-line. To accomplish this efficiently, we assume that we have already computed $\pi(\beta)$ for each blue half-line $\beta$ as described in Section 3.2.1.

To compute $|\overline{\mathcal{B}}(\rho)|$, we sweep a vertical line from right to left (refer to Figure 6 for an illustration). During the sweep we maintain a balanced binary search tree (BST) $\mathcal{T}$ on the slopes of $\pi(\beta)$. That is, when the sweep line encounters an endpoint of a blue half-line $\beta$ and $\pi(\beta)$ is defined, we insert the slope of $\pi(\beta)$ into $\mathcal{T}$. If $\pi(\beta)$ is undefined, then there is no red half-line below the endpoint of $\beta$ and since each red half-line $\rho$ is defined for all $x \geq \rho_{x}$, the endpoint of $\beta$ does not lie above any red half-line and can be safely ignored.

At time $\rho_{x}$ of the sweep, that is, when the sweep line encounters a red half-line endpoint $\rho_{x, y}$, the number of entries in $\mathcal{T}$ that are greater than or equal to the slope of $\rho$ is equal to the number of blue half-line endpoints above $\rho$. To see this, observe that when $\rho_{x}$ is encountered, tree $\mathcal{T}$ contains all blue half-line endpoints that have a well-defined above-below relationship with $\rho$. Since the red half-lines do not intersect other red half-lines, the ordering of the slopes of the red half-lines is equivalent to the above-below relationship among the red half-lines that are defined at $\rho_{x}$. The above-below relationship between red half-lines and blue half-line endpoints defines a partial order, which means that if $\beta_{x, y}$ is above $\rho_{1}$, then both $\rho_{1}$ and $\rho_{2}$ are defined at $\beta_{x}$ and $\rho_{1}\left(\beta_{x}\right)>\rho_{2}\left(\beta_{x}\right)$, then $\beta_{x, y}$ is also above $\rho_{2}$. Consequently, the set of endpoints of blue half-lines 

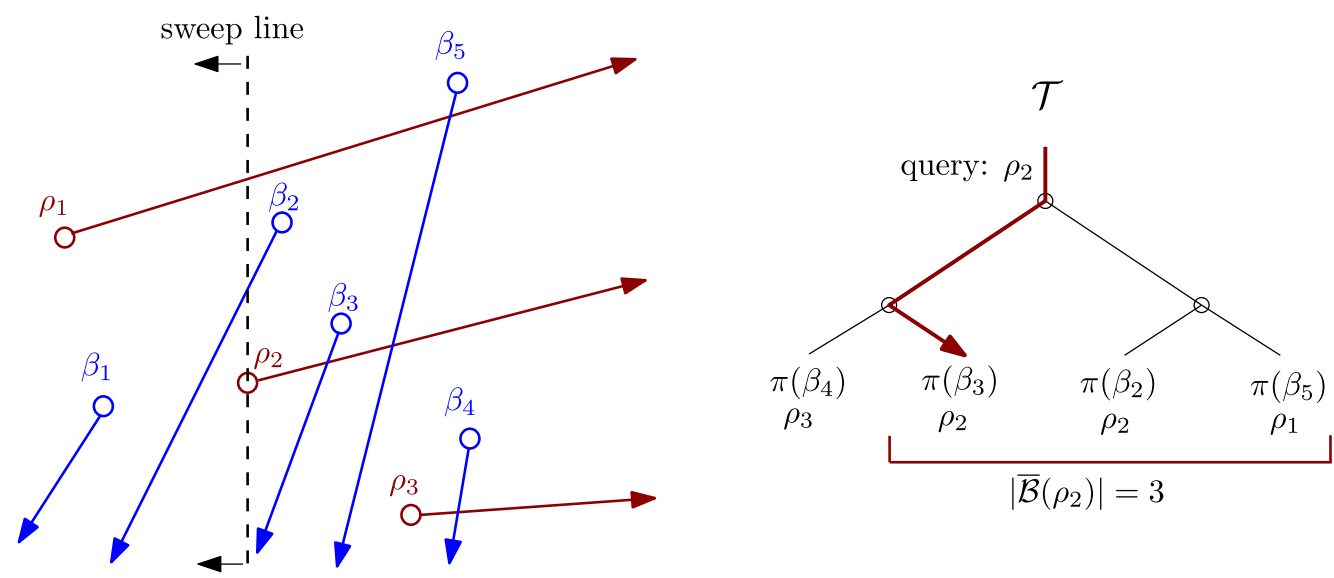

Fig. 6. Example of the plane sweep algorithm used to find $|\overline{\mathcal{B}}(\rho)|$. The vertical sweep line moves from right to left and, when a blue endpoint $\beta_{x, y}$ is encountered, $\pi(\beta)$ is added to the search tree $\mathcal{T}$. When the sweep line encounters a red endpoint $\rho_{x, y}$, the tree is queried by the slope of $\rho$. The number of leaves in the search tree that have slopes greater than or equal to the slope of $\rho$ is equal to $|\bar{B}(\rho)|$.

above $\rho$ is equal to the set of blue half-lines $\beta$ with slopes of $\pi(\beta)$ greater than the slope of $\rho$. See Figure 6 for an illustration of this plane sweep.

Given the above, whenever the sweep line encounters an endpoint of a red half-line $\rho$, we perform predecessor/successor query on $\mathcal{T}$ using the slope of $\rho$ to find the number of points above $\rho$. Maintaining and querying $\mathcal{T}$ takes $O(\log n)$ time per blue half-line endpoint (insertion) or red half-line endpoint (query), resulting in $O(n \log n)$ time overall to compute $|\bar{B}(\rho)|$ for each half-line $\rho$.

\subsection{Maintaining Critical Rays}

Our overall divide-and-conquer algorithm relies on the knowledge of the critical rays at the beginning of each recursive call. At the base case, subset $P[l: r]$ contains only one point. Therefore, both left and right critical rays of that point are directed vertically downward. Thereafter, at the end of each recursive call, we update these rays by recomputing only the right critical ray for each point in $P[l: k]$ and the left critical ray for each point in $P[k+1: r]$. To do this, we need the next lemma.

Lemma 3.5. The tangent from $p_{i} \in P[l: k]$ to the upper convex hull of all vertices in $P[k+1: r]$ is the critical ray $c_{\text {right }}\left(p_{i}, P[l: r]\right)$ if and only if the vertex $p_{t}$ on the hull that the tangent goes through is visible to $p_{i}$. Symmetrically, the tangent $p_{j} \in P[k+1: r]$ to the upper hull of vertices in $P[l: k]$ is the critical ray $c_{\text {left }}\left(p_{j}, P[l: r]\right)$ if and only if the tangent point $p_{t^{\prime}}$ on the hull is visible to $p_{j}$.

Proof. We first prove that only points on the upper hull of $P[k+1: r]$ can be candidates for defining $c_{\text {right }}\left(p_{i}, P[l: r]\right)$. Suppose that $p_{t}$ is the point in $P[k+1: r]$ that defines $c_{r i g h t}\left(p_{i}, P[l: r]\right)$ and that $p_{t}$ does not fall on the upper convex hull of $P[k+1: r]$. By definition, no point in $P[k+1: r]$ can fall outside the upper hull of the same point set, and therefore $p_{t}$ must fall inside the hull. In that case, the ray that starts from $p_{i}$ and goes through $p_{t}$ intersects the upper hull of $P[k+1: r]$. Let $p^{\prime}$ be this intersection point, and let $p^{\prime \prime}$ be the vertex of the upper hull that is exactly to the right of $p^{\prime}$. Then $p^{\prime \prime}$ is visible from $p_{i}$, which contradicts the assumption that $p_{t}$ defines $c_{\text {right }}\left(p_{i}, P[l: r]\right)$. 
Let $p_{t}$ be the point on the upper hull, such that the tangent goes through $p_{t}$. If $p_{t}$ is not visible to $p_{i}$, then there is a point $p_{m}$ s.t. $i<m<t$ that is above the visibility ray $\overrightarrow{p_{i} p_{t}}$. Since the tangent from $p_{i}$ to the upper hull goes through $p_{t}, p_{m}$ must not be in the set encompassed by the upper hull. Therefore, $p_{m}$ is in the set included with $p_{i}$ and the previous critical ray of $p_{i}$ has a larger slope than the tangent, so the tangent is not $c_{\text {right }}\left(p_{i}, P[l: r]\right)$.

If, however, $p_{t}$ is visible to $p_{i}$, then $c_{\text {right }}\left(p_{i}, P[l: k]\right)$ falls below $p_{t}$. Furthermore, the visibility ray from $p_{i}$ to other points on the upper hull are below the tangent (by property of tangents) and therefore the tangent is the only visibility line that is not below any other point of the upper hull. Hence the tangent is $c_{\text {right }}\left(p_{i}, P[l: r]\right)$. The proof involving $c_{\text {left }}\left(p_{j}, P[l: r]\right)$ is symmetric.

Thus, to update $c_{\text {left }}\left(p_{i}, P[l: r]\right)$ and $c_{\text {right }}\left(p_{i}, P[l: r]\right)$ we utilize the upper convex hulls of $P[l: k]$ and $P[k+1: r]$ (computed in the previous recursive step), and for every point in these two subsets we construct the tangent to the hull of the opposite subset. To construct the upper convex hull of $P[l: r]$ for the next recursive step, we simply merge our two upper hulls in $O(n)$ time. Computing tangents is equivalent to binary searches, which takes $O(\log n)$ time per tangent for a total of $O(n \log n)$ time. Combining this with the rest of the analysis presented in Section 3, we conclude that we can solve each recursive level of 1 DVisibilityIndex in $O(n \log n)$ time. Hence, the total running time of algorithm 1DVISIBILITYINDEX is $O\left(n \log ^{2} n\right)$.

\section{PARALLEL EXTENSION}

In Section 3.2 we present a plane sweep-based solution to the red-blue line segment intersection problem, that we perform at each stage of Bipartite Visibility. This solution operates by maintaining a tree structure while sweeping across all points. In the example in Figure 6, the tree contains all blue rays that intersect the sweep line and, when a red endpoint is encountered, it is queried into the tree. Since the tree structure varies as the sweep line moves through the set of points, this approach is highly sequential. However, if we can construct a structure that contains all versions of the tree, we can perform all queries in parallel. We note that this is performed at each stage of Bipartite Visibility and, since critical rays are updated, we do not re-use structures.

Persistence [8] is a technique for efficiently maintaining all past versions of a dynamic structure for future queries. A persistent binary search tree (BST) supports all standard update operations and maintains information about previous versions of the search tree, allowing queries to be performed on any past version (as though subsequent updates had not been performed. In this work, we consider the offline construction of persistent BSTs, where we assume that all updates are provided up-front, allowing us to build a static search tree. Given an ordered set $O$ of queries and updates, we construct a persistent BST, incorporating all updates $U \subset O$ into the data structure. We can then perform each query, $q \in O$, on the version of the BST corresponding to all updates that precede $q$ in the set $O$. Each $q \in O$ can be performed independently once the persistent BST is constructed. Thus, if we construct a balanced persistent BST, $n$ queries can be answered in parallel in $O(\log n)$ time using $n$ processors, i.e., in $O(n \log n)$ work in the CREW PRAM model. Offline persistent BSTs can be used to parallelize algorithms that rely on plane sweep algorithms. In this section we detail the two offline persistent BST data structures that we use to solve our red-blue line segment intersection problem (and thus solve Bipartite Visibility). Note that, for each plane sweep operation performed, we first sort endpoints of half-lines by their x-coordinate (in $O(\log n)$ time and $O(n \log n)$ work), which is required for both the plane sweep algorithm and construction of a persistent search tree. Furthermore, all other operations performed by our divide-and-conquer algorithm (described in Section 3) can be easily parallelized: all $n$ critical rays can be updated concurrently in $O(\log n)$ time and, using these critical rays, we can merge upper convex hulls in $O(1)$ time and $O(n)$ work. 
If we can implement the search tree used in the plane sweep of Section 3 as an offline persistent BST, then we can perform the sweep in $O(\log n)$ time and $O(n \log n)$ work. Thus, the parallel runtime and work of the overall algorithm can be defined by the recurrences $\Phi(n)=\Phi(n / 2)+$ $O(\log n)=O\left(\log ^{2} n\right)$ and $W(n)=2 W(n / 2)+O(n \log n)=O\left(n \log ^{2} n\right)$, respectively. This yields the following theorem.

THEOREM 4.1. The $1 D$ total visibility-index problem can be solved in $O\left(\log ^{2} n\right)$ time and $O\left(n \log ^{2} n\right)$ work in the CREW PRAM model.

The work complexity of the parallel algorithm matches our sequential algorithm runtime, which is the best we can hope for from a parallel algorithm.

We identify two offline persistent BST data structures that we can use to solve Bipartite Visibility in parallel. In Section 4.1 we present an overview of these data structures and in Section 4.2 we describe some relevant details of our implementations that use these persistent data structures.

\subsection{Overview of Persistent BST Structures}

In this subsection, we provide overviews of two offline parallel BST data structures that we employ: the array-of-trees [3] and the linear-space persistent BST [6]. Here we present an overview of these two data structures and we refer interested readers to References [3] and [6] for further details.

Array-of-trees. Atallah et al. [3] describe a data structure that they call array-of-trees, which implements a persistent search tree and can be built in the CREW PRAM model in $O(\log n)$ time and $O(n \log n)$ work. Hence, we can use an array-of-trees to implement the tree that is maintained during the plane sweep described in Section 3.2. Thus enables us to perform the plane sweep operation in $O(\log n)$ parallel time and $O(n \log n)$ work.

Our first parallel implementation replaces each plane sweep operation described in Section 3.1 with the construction and querying of an array-of-trees (AoT). AoTs are constructed by starting with the input data as a set of elements (key $k$, time $t$ pairs), sorted by $k$. This initial dataset becomes the leaf level of the AoT, with each element corresponding to a node. The AoT is constructed bottom-up by merging pairs of nodes to create parent nodes. Each non-leaf node contains a list of elements contained in its children, sorted by $t$ (e.g., the root contains the input data sorted by $t$ ). For each element, each node also maintains a pointer to the element in each child node with largest $t_{c h i l d}$, s.t., $t_{c h i l d} \leq t$. Thus, we can consider any element $e=\left(e_{k}, e_{t}\right)$ as a node in a BST that can be searched by key $k$, but only contains the elements with $t \leq e_{t}$.

Querying the AoT involves two steps: (1) finding the correct element in the root (by $t$ ) and (2) querying the corresponding BST (by $k$ ). Given a query $q=\left(q_{k}, q_{t}\right)$, the correct root element is found by performing a binary search using $q_{t}$. The corresponding element in the root acts as the root of a BST that we then search by $q_{k}$ (by following pointers down the AoT). Each of these two steps requires $O(\log n)$ work per query and replacing a plane sweep operation requires $O(n)$ such queries. Thus, an AoT can be used in place of a plane sweep and requires $O(\log n)$ time and $O(n \log n)$ work in the CREW PRAM model.

While the AoT structure can be constructed simply and allows for easy parallelization, its primary drawback is the space requirement. At each level of the structure, $O(n)$ elements are stored, so the total structure requires $O(n \log n)$ space. When using an AoT to replace plane sweep operations on a large dataset, the memory requirement may become detrimental to overall performance.

Linear-space Persistent BST. To avoid the $O(n \log n)$ space requirement of the array-of-trees, we consider a more complex data structure. Chazelle and Edelsbrunner [6] present a technique to solve some types of range queries using only $O(n)$ space in the word-RAM model [16]. Recall that the AoT data structure, described above, allows querying at any time $t$ by storing $O(n)$ key values (and pointers) at each level. The linear-space persistent BST (LPBST) presented in Reference [6], 


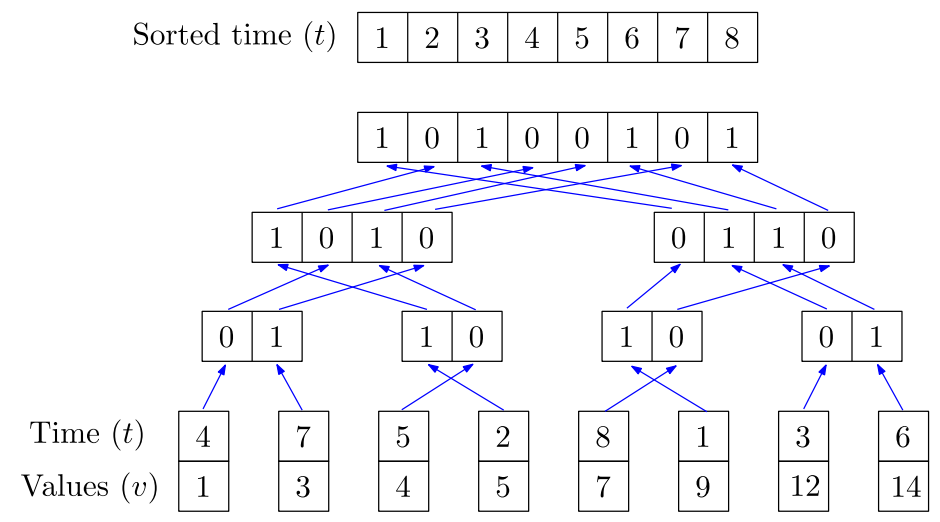

Fig. 7. Illustration of how a LPBST is constructed.

however, stores only $O(n)$ bits at each level, resulting in a total space requirement of $O\left(\frac{n \log n}{w}\right)$, where $w$ is the number of bits that fit in a machine word. If we assume a constant number of duplicate values, then $w=\Theta(\log n)$. Thus, a LPBST structure requires only $O(n)$ space.

The process of constructing a LPBST is similar to that of an array-of-trees. An input of pairs (key $k$, time $t$ ), sorted by key $(k)$ is provided as input. As with an AoT, the LPBST structure can be built bottom-up by merging pairs of elements, resulting in sublists sorted by $t$. However, unlike AoTs, we do not store copies of $k$ and $t$ values at each node: "nodes" of a LPBST store only a single bit per merged element to identify which child list the element came from. A 0 (respectively, 1) bit is stored if the element was merged from the left,(respectively, right) list. Thus, to construct the LPBST, we merge pairs of nodes (as we do when constructing the AoT); however, we store only bits in the LPBST structure and delete the intermediate $k$ and $t$ values that were used to perform the merge. This merging process is repeated until all $O(\log n)$ levels are merged. At the final (root) level, we do not delete the $k$ and $t$ merge values and maintain the final list sorted by $t$. As illustrated in Figure 7, the final LPBST contains a sorted list of $t$ values at the root, $O(n \log n)$ bits, and the initial $(k, t)$ pairs as leaf nodes. Note that since each bit within a node represents which subtree (left or right) a particular value came from, the total number of 0 or 1 bits represents the sizes of a nodes' left and right subtrees, respectively.

Given a query $\left(q_{k}, q_{t}\right)$, we can use the LPBST to find the number of elements that have time $t \leq q_{t}$ and key $k \leq q_{k}$. Querying involves first finding the number of elements with $t \leq q_{t}$ by finding $\operatorname{rank}\left(q_{t}\right)$ : the index of $q_{t}$ in the sorted list of $t$ values at the root. Any entry in the root node with index $i>\operatorname{rank}\left(q_{t}\right)$ has time greater than $q_{t}$. Thus, we query the LPBST structure, concerned only with bits of index $i \leq \operatorname{rank}\left(q_{t}\right)$. We determine the number of elements in the left and right subtrees, $s u b_{L}$ and $s u b_{R}$, by counting the number of $0 \mathrm{~s}$ and $1 \mathrm{~s}$ in the array of bits at the root (with index $\left.i \leq \operatorname{rank}\left(q_{t}\right)\right)$. Since the LPBST is a BST on keys, the query process simply traverses the tree while counting bits to determine $s u b_{L}$ and $s u b_{R}$ at each node. These subtree sizes are used to count the total number of query matches. Figure 8 provides an example illustrating how a query is performed on a linear-space persistent BST.

Computing $s u b_{L}$ and $s u b_{R}$ for a given node is accomplished by performing a prefix sums operation on the bits contained in the node. While scanning a node may take $O(n)$ time, Chazelle and Edelsbrunner [6] reduce the time to compute prefix sums on the list of bits in a node by storing the partial prefix sums every $\log n$ bits. This only requires an additional $O(n)$ space and, using a lookup table to count bits within a word of $\log n$ bits, allows queries to be performed in $O(\log n)$ 


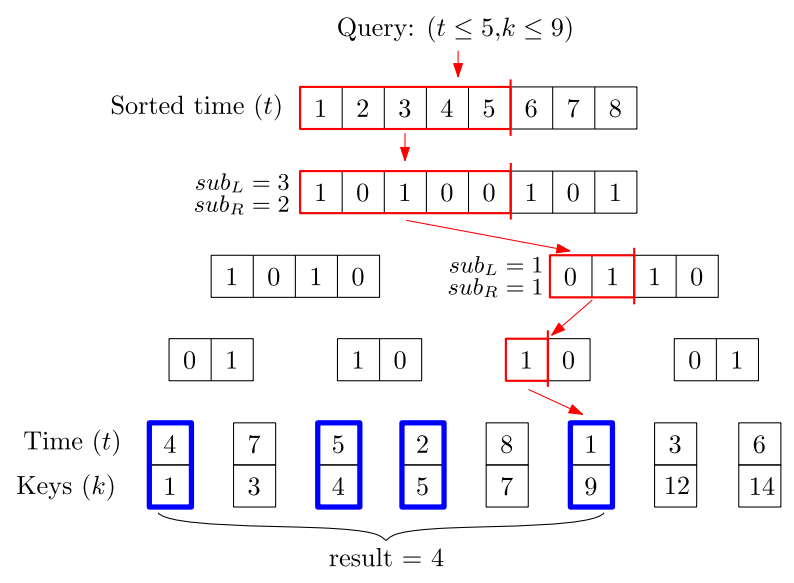

Fig. 8. Example of a query being performed on a LPBST.

time. Thus, the LPBST can be used in place of some plane sweep operations and requires $O(\log n)$ time, $O(n \log n)$ work, and $O(n)$ additional space.

\subsection{Implementation Details}

Aside from our implementation of the NAIVE algorithm, all of our implementations employ the divide-and-conquer approach described in Section 3. At each recursive level, we perform a total of $O(n \log n)$ work. However, the size of each independent task depends on the recursive level (e.g., at the lowest level, we determine visibility between pairs of vertices). Therefore, at low levels of recursion, our parallel implementations are able to concurrently perform each task without requiring parallelization. At the top level of recursion, however, we have a single task that must be executed in parallel. Thus, our parallel implementations attempt to avoid parallelization overhead by dynamically parallelizing tasks only when necessary at the top levels of recursion.

Both the construction and querying of AoTs and linear-space persistent BSTs are similar in many ways. Therefore, our implementations of these structures use many of the same methods. We construct both structures bottom-up by merging pairs of sublists while storing resulting values (or bits). To perform this merging in parallel, we employ the techniques outlined in Reference [19] to merge two lists in $O(\log n)$ time and $O(n)$ work. However, when constructing LPBSTs, efficiently storing bits requires careful consideration. On modern CPUs, the smallest addressable unit of memory is a byte ( 8 bits). Thus, if we define each bit independently (e.g., as a Boolean datatype), each bit will require 8 bits of storage space. To avoid wasting space, we use bitwise operations to manually pack bits of data into words of $w$ bits each. We leave $w$ as a parameter and empirically measure the ideal configuration for our hardware platforms.

While the querying process of these two structures is also similar, querying a LPBST requires computing the prefix sums of bits at each node. For our implementation, we store partial prefix sums every $w$ elements. However, while Chazelle and Edelsbrunner [6] use lookup tables to count bits within words of $\log n$ bits, we employ the popcount hardware operation. popcount is available on our hardware platforms (described in Section 5.1) and returns the number of 1 bits in a word. Since variations of popcount are available for words of $8,16,32$, and 64 bits, our choice of $w$ is limited to these options.

Since our implementation stores a total of $\frac{n}{w}$ partial prefix sum values, our choice of $w$ dictates our space requirement. Furthermore, depending on the details of the popcount operation, $w$ may 


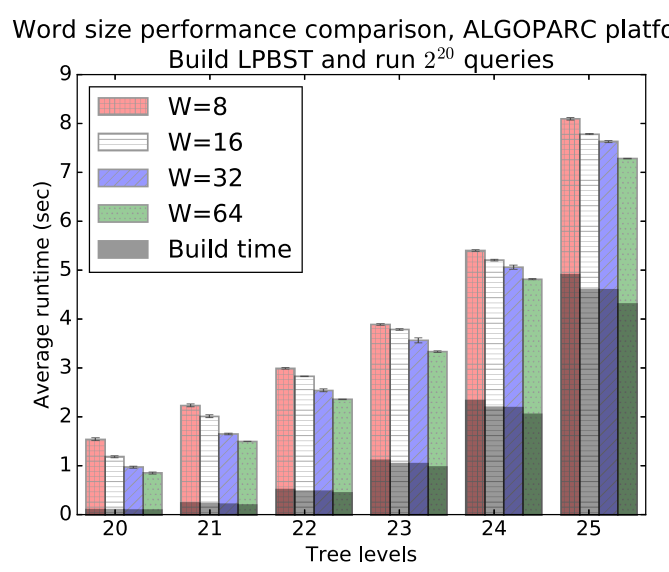

Fig. 9. Average runtime to construct a LPBST and perform $2^{20}$ queries. The dark shaded portion of each bar indicates construction time, while the remaining time is spent querying.

impact query performance. To determine the ideal $w$ value for our hardware platforms, we measure the relative query and construction performance while varying $w$ on a range of synthetic, random datasets (see Section 5.2 for details on dataset construction). Figure 9 contains the average runtime to build a LPBST and perform $2^{20}$ queries on it on the ALGOPARC platform (detailed in Section 5.1). Results indicate that $w=64$ provides the best performance for our hardware. This is not surprising, since smaller $w$ values require that we store more partial prefix sums. We use $w=64$ for all experiments hereafter unless otherwise noted. We note that larger $w$ values may further improve performance, but popcount is not available for larger word sizes and lookup tables would be far too large to be practical.

\section{EXPERIMENTAL RESULTS}

In this section we present an empirical evaluation of the performance of our algorithms on synthetic and real-world datasets. We develop five implementations: NaIve, RedBlue, Sweep, PARAoT, and LinPar. NAIVE implements the $O\left(n^{2}\right)$ algorithm described in Section 3 and is used as baseline. RedBlue, Sweep, PArAot, and LinPar all use the divide-and-conquer approach presented in Section 3 but they differ in the implementation of the half-line intersection counting step: RedBLue implements the Palazzi and Snoeyink [23] algorithm for red-blue line segment intersection counting, SwEEP implements the algorithm presented in Section 3 using plane sweep, PARAoT employs the array-of-trees data structure described in Section 4, and LinPAR uses the linear-space structure, which is also described in Section 4. Asymptotically, all four algorithms achieve $O\left(n \log ^{2} n\right)$ sequential running time. However, RedBLuE is more complex than our other implementations and has the poorest performance in practice among our non-naive implementations. While all five implementations run sequentially, PARAoT and LinPAR can also run in parallel mode, using multiple threads to improve performance. Though they are both amenable to parallelization, PARAOT requires more memory, while LINPAR is more complex and relies on the hardware-specific popcount operation.

\subsection{Methodology}

All algorithms are implemented in $\mathrm{C}++$ and compiled with gcc 4.8.5 using the -Ofast optimization flag. Parallel execution is performed using the openMP library that is included with the gcc 


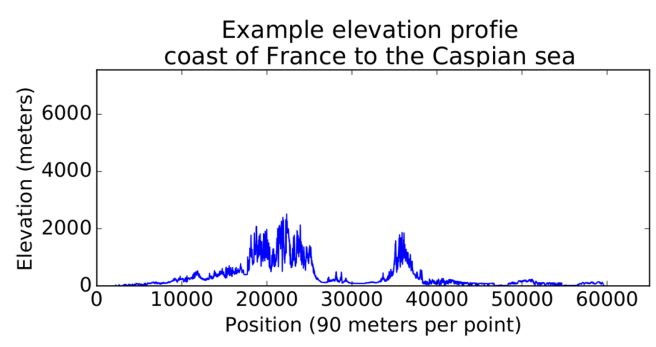

(a) Example elevation profile from Europe.

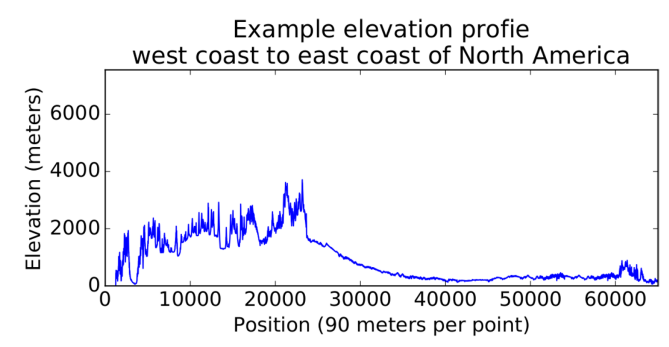

(b) Example elevation profile from North America.

Fig. 10. Examples of elevation profiles from $2^{16}$-point slices of the Earth dataset (note the different scales).

compiler. All geometric structures, predicates, and primitives used by all of our algorithms are custom implementations. ${ }^{1}$

We use two hardware platforms for our evaluation. The 4-core AlgOPARC platform is comprised of an Intel Xeon E5-1620 processor (4-core, $3.6 \mathrm{GHz}$ ) and $16 \mathrm{GiB}$ of RAM, running the Ubuntu 16.04 operating system. Algoparc has hyperthreading enabled, providing 8 virtual cores. The 20 -core UHнPC platform is comprised of two Intel Xeon E5-2680 processors (10-core, $2.80 \mathrm{GHz}), 128 \mathrm{GiB}$ of RAM, and runs the Red Hat Server 6.5 operating system. Note that UHHPC has 2 CPU sockets, each with four memory channels to RAM and an independent L3 cache. All experimental results are averaged over 10 iterations with error bars shown when significant.

\subsection{Datasets}

We evaluate our algorithm implementations on three synthetic datasets. We consider a flat dataset in which all points' elevations are set to $h_{i}=1$, so that each point can only see its (at most two) neighboring points. For this dataset, RedBlue, Sweep, ParAoT, and LinPar compute few intersections at each level of recursion, and thus provides a simple correctness case and a performance baseline. We consider a parabolic dataset in which each point's elevation is set to $h_{i}=i^{2}$, so that every point can see every other point. For this dataset, our four recursive implementations compute many intersections at each level of recursion. Finally, we consider Random datasets in which point elevations are uniformly sampled from the range $\left[1,10^{6}\right]$.

We also perform evaluations on datasets generated from real-world terrain maps. The CGIARCSI Global-Aridity and Global-PET Database [29,30] consists of elevation data for the entire Earth with $90 \mathrm{~m}$ resolution. We extract one-dimensional slices from four different regions: Europe, Asia, Africa, and North America. Each slice consists of $2^{16}$ points (spanning $\sim 5000 \mathrm{~km}$ ). For each of the four regions, we extract 10 East-West slices at $1 \mathrm{~km}$ North-South intervals to provide a diverse set of datasets based on real-world terrains. While the different slices taken from a single region vary only slightly, the different regions lead to diverse elevation maps, as seen in Figure 10.

\subsection{Sequential Performance Results}

We evaluate our sequential implementations and sequential execution of PARAoT and LinPAR (i.e., using 1 thread) on the Algoparc platform. Figure 11 shows average runtime vs. dataset size $(n)$ for synthetic random datasets. As expected, the quadratic complexity of NAIVE results in much sharper runtime growth as we increase $n$, compared to the $O\left(n \log ^{2} n\right)$ algorithms. Additionally,

\footnotetext{
${ }^{1}$ For simplicity, our implementations use 64-bit double-precision floating point variables, ignoring potential inaccuracies arising from round-off errors. If necessary, such inaccuracies can be avoided via exact arithmetic, e.g., as implemented in the CGAL library [25].
} 


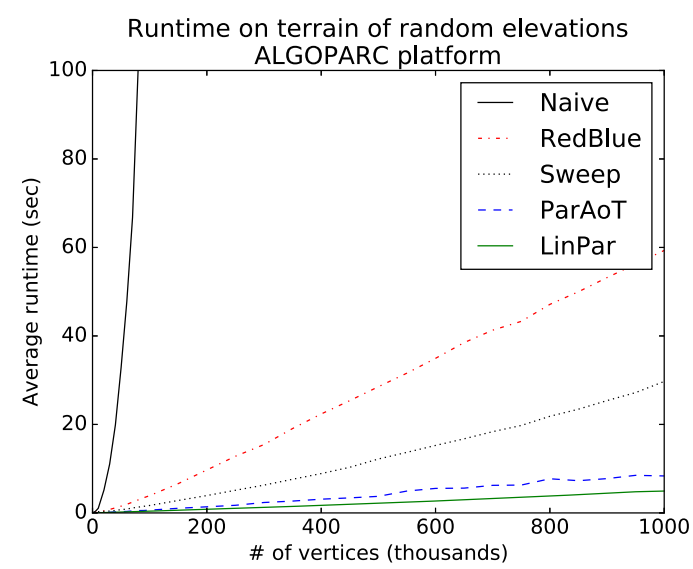

Fig. 11. Results with all five implementations for the Random dataset.

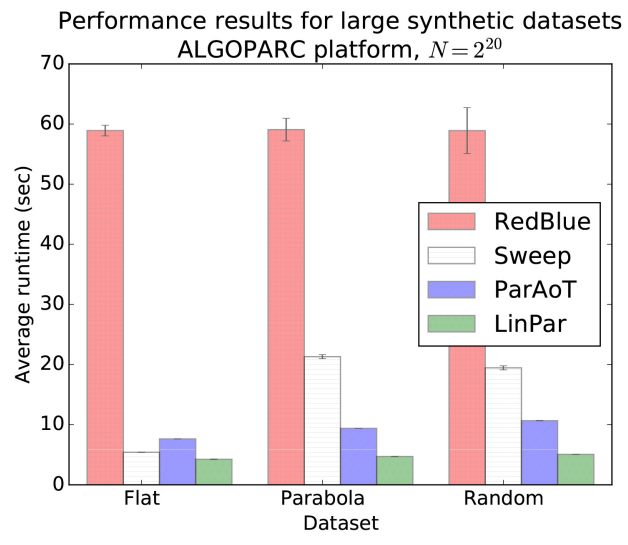

(a) Performance on synthetic datasets $\left(n=2^{20}\right)$.

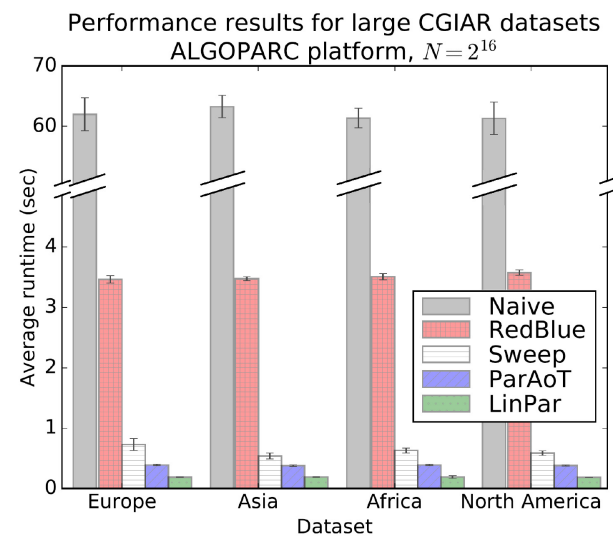

(b) Performance on real-world datasets $\left(n=2^{16}\right)$.

Fig. 12. Sequential Performance of our five implementations.

we see that the simplified half-line intersection counting algorithm described in Section 3.1 gives Sweep, PARAoT, and LinPar a significant practical performance advantage over RedBlue. Note that, to compare sequential performance, we run PARAoT and LiNPAR using only one thread.

Figure 12(a) shows average runtimes of our four sub-quadratic implementations for our three classes of synthetic datasets of $n=2^{20}$ vertices (we omit NAIVE results, since its runtime is prohibitive for such a large $n$ ). These results confirm that SwEEP, PARAoT, and LinPAR are consistently faster than REDBLUE, with an average decrease in runtime (across all synthetic inputs) of 5.56×, $6.51 \times$, and $12.70 \times$, respectively. Figure $12(\mathrm{a})$ further reveals that SwEEP has a significant variance in execution time across different synthetic datasets, indicating that the overhead of maintaining and balancing a large BST during the plane sweep has a major impact on algorithm performance. The performance of PARAOT and LiNPAR, however, are not as dependent on the dataset, and they therefore outperform SwEEP on all but the flat synthetic datasets. LINPAR is our fastest implementation on all synthetic datasets, decreasing runtime over PARAoT by $1.79 \times, 1.99 \times$, and $2.11 \times$ on flat, parabolic, and random inputs, respectively. 


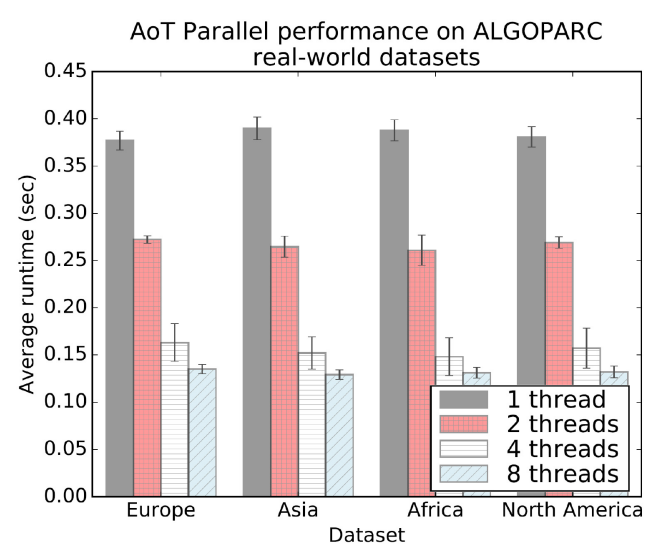

(a) Algoparc platform.

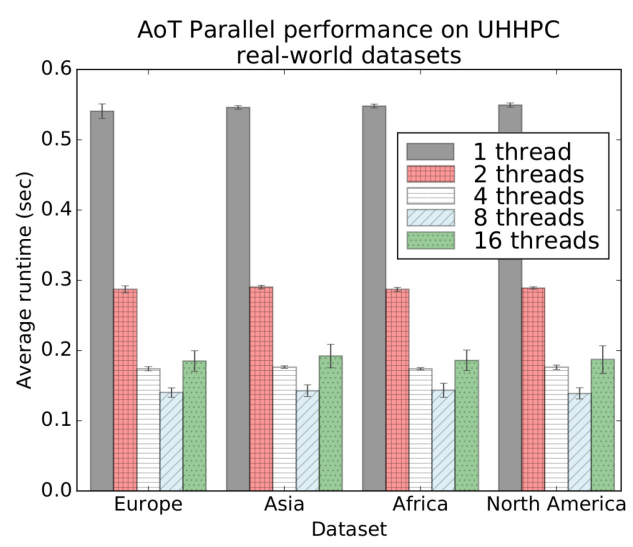

(b) UннРс platform.

Fig. 13. Parallel performance of the PARAoT implementation on real-world datasets for varying number of compute threads on each of our two hardware platforms.

Figure 12(b) shows runtimes for each algorithm when applied to data from each region of our real-world dataset, averaged over all 10 slices. As with synthetic datasets, SwEEP, PARAoT, and LINPAR greatly outperform REDBLUE with an average decrease in runtime of $5.72 \times, 8.25 \times$, and $18.69 \times$, respectively. We conclude that our simplified half-line intersection algorithm provides a significant performance improvement over the general red-blue line segment intersection counting algorithm [23] used by RedBlue. Furthermore, even sequentially, PARAoT and LinPAR are faster and more consistent that SwEEP, indicating that the AoT and LPBST data structures provide an effective alternative to plane sweep for this problem. Additionally, LiNPAR has a significant performance advantage over all of our other implementations, indicating that the reduced memory usage of LINPAR provides practical performance gains.

\subsection{Parallel Performance Results}

In addition to providing the performance benefits seen in the results above, the AoT and LPBST data structures are amenable to parallelization. In this section, we evaluate the performance of our parallel implementations of PARAoT and LinPAR.

To assess parallel performance from a practical standpoint, we present results obtained using our real-world datasets. Figure 13 and Figure 14 show the average runtime of PARAoT and LinPAR, respectively, using our real-world datasets for various numbers of threads on our two hardware platforms. While parallelization provides some performance improvement, the speedup is far from the peak, especially as the number of threads increases. On the four-core ( 8 virtual cores due to hyperthreading) ALGOPARC platform, PARAoT and LinPAR achieve a maximum speedup of 2.92 and 4.24, respectively, with 8 threads. On the UHHPC platform, however, PARAoT and LinPAR achieve a maximum parallel speedup of 3.86 and 7.39, respectively. Furthermore, while LINPAR achieves its maximum speedup with 16 threads, PARAoT achieves its maximum parallel speedup with only 8 threads and slows down when using 16 threads. We note that our real-world datasets contain only $2^{16}$ data points. On such small datasets, the affect of the cache system may significantly impact parallel performance. Both of our hardware platforms have L3 caches that can store more than $2^{16}$ elements (ALGOPARC and UHHPC have L3 caches of $10 \mathrm{MB}$ and $25 \mathrm{MB}$, respectively). This results in very fast sequential execution, limiting the achievable parallel speedup and increasing the relative cost of parallel overhead (e.g., spawning new threads). We speculate that the large memory 


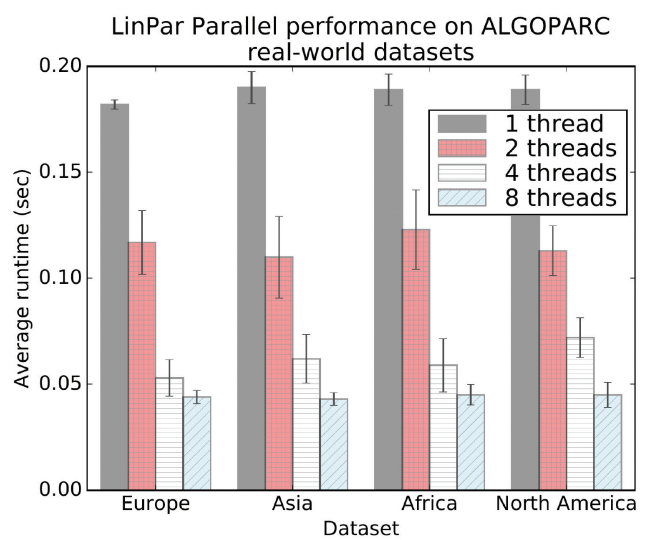

(a) Algoparc platform

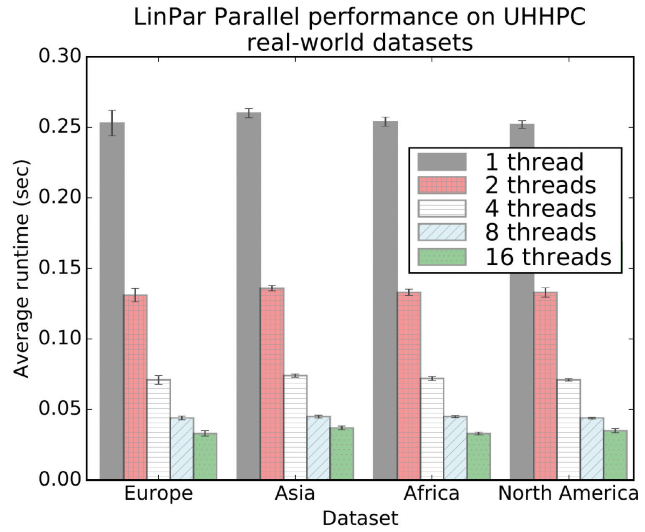

(b) UннPC platform

Fig. 14. Parallel performance of the LINPAR implementation on real-world datasets for varying number of compute threads on each of our two hardware platforms.

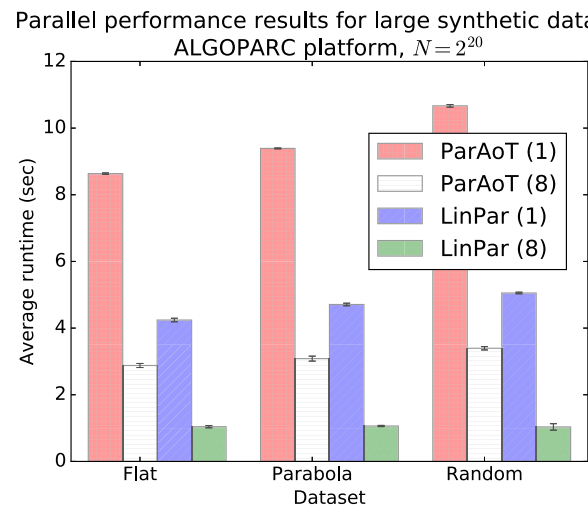

(a) Algoparc runtime.

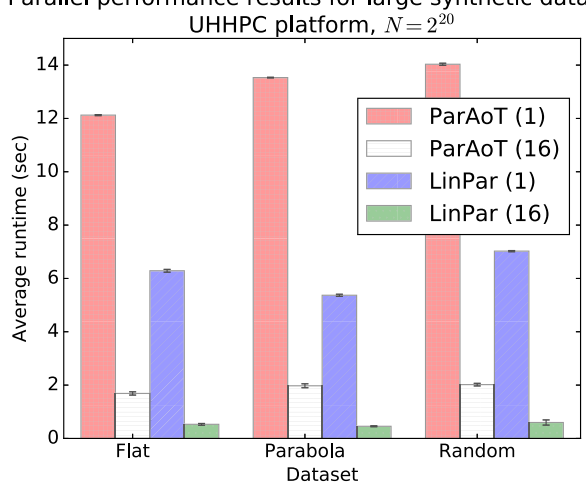

(b) UHHPC runtime.

Fig. 15. Average runtime of our parallel implementations on synthetic datasets $\left(n=2^{20}\right)$ on each of our platforms. Number of threads used shown in parenthesis.

requirement of the AoT data structure further increases the impact of the cache systems on parallel speedup, resulting in the decrease in performance when using 16 threads on the UHHPC platform.

To better understand the cause of the limited parallel performance on real-world datasets, we perform a series of experiments on synthetic random datasets (for which we can vary the size) using the (empirically) best number of threads for each hardware platform. Figure 15 plots the average sequential and parallel execution time of PARAoT and LINPAR on varying synthetic datasets, for each of our hardware platforms. Note that these datasets are the same used in our sequential experiments (Figure 12(a)). Figure 16 shows the average parallel speedup vs. dataset size on each hardware platform for PARAoT and LinPAR. For $n=2^{16}$ vertices, parallel performance results are similar to our real-world results. As the dataset size increases, however, parallel speedup increases for both implementations, on both hardware platforms. At the largest input size PARAoT can process (due to memory requirements), we see a maximum speedup of 3.51 and 7.25 on AlgopARC and UHHPC, respectively. PARAoT's parallel speedup remains well below expected 


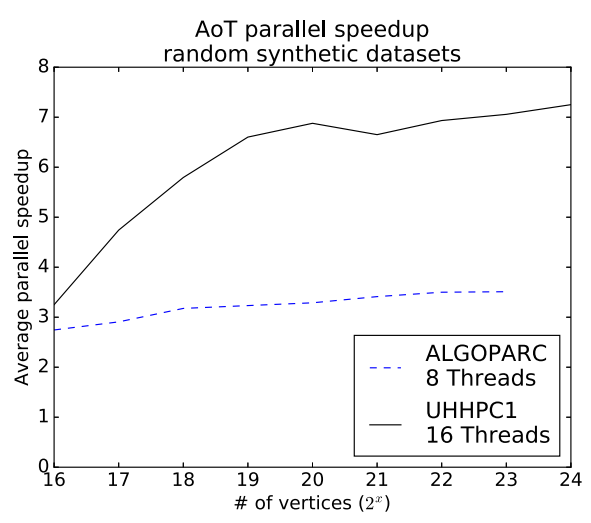

(a) PARAoT parallel speedup.

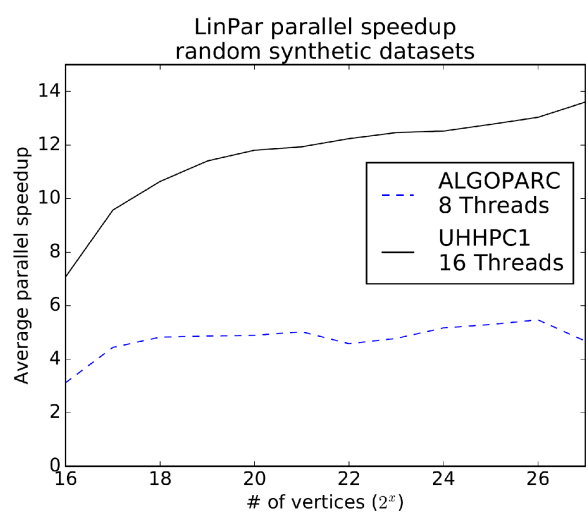

(b) LiNPAR parallel speedup.

Fig. 16. Average parallel speedup obtained on random inputs of varying size. Note that axis scales differ.

parallel performance, especially for UHHPC, where we would expect a speedup nearing 16 for 16 threads. LINPAR, however, achieves significantly higher parallel speedup, with a maximum of 5.46 and 13.60 on AlgopARC and UhHPC, respectively. We note that the performance drop seen in Figure 16(b) when $n=2^{27}$ on Algoparc is due to limited memory, causing the system to begin swapping to disk. On UHнPC, however, we have much more available memory, and we see that LiNPAR continues to gain additional parallel speedup as we increase the input size.

As discussed in Section 4, the array-of-trees data structure requires $O(n \log n)$ memory, while LPBST requires only $O(n)$ additional space. The results in Figure 16 suggest that this memory requirement may be causing PARAoT to be memory bound, resulting in a memory bandwidth bottleneck that limits parallel speedup. This is supported by the fact that the parallel speedup obtained by PARAoT on each hardware platform corresponds to the number of available memory channels. Algoparc, while running 8 hardware threads, is limited by four memory channels and achieves a maximum speedup of 3.51. UHнPC has eight memory channels (four per socket) and achieves a maximum speedup of 7.25, despite using 16 hardware threads. We speculate that LinPAR, requiring only linear additional space, does not suffer from this memory bottleneck and is therefore able to achieve much higher parallel speedup. We conclude that LINPAR is our fastest implementation, both sequentially and in parallel.

\section{CONCLUSIONS}

In this work, we presented an $O\left(n \log ^{2} n\right)$ algorithm to solve the $1 D$ total visibility-index problem. Our divide-and-conquer approach uses dualization to reduce the problem to a series of instances the red-blue line segment intersection counting problem. To the best of our knowledge, this is the first subquadratic-time algorithm to solve this problem.

We implemented four versions of this algorithm and evaluated their performance on two distinct hardware platforms. Each of our implementations solves the red-blue line segment intersection counting problem differently: REDBLuE relies on an existing general-case solution, SweEP uses a plane sweep algorithm, and PARAoT and LINPAR employ persistent search tree data structures. While all four implementations have $O\left(n \log ^{2} n\right)$ asymptotic runtime and are at least an order of magnitude faster than the naive $O\left(n^{2}\right)$ solution, their relative performance differs greatly. Empirical results show that REDBLUE is, on average, at least 5 times slower than our other three implementations, indicating that our special-case red-blue line segment intersection counting technique 
provides significant performance gains. Furthermore, our two implementations that rely on persistent data structures out-perform our plane sweep implementation on most synthetic and all real-world datasets.

In addition to sequential performance gains over other implementations, PARAoT and LiNPAR can leverage multiple threads to further improve performance. While both implementations achieve parallel speedup on both of our hardware platforms, PARAoT's performance gains are limited due to its large memory requirement. LINPAR, however, requires only $O(n)$ space and, therefore, achieves up to $85 \%$ parallel efficiency on large synthetic datasets. Our fastest implementation finds the $1 \mathrm{D}$ total visibility-index of over 100 million vertices in under 3 minutes using 16 threads.

An interesting open problem is to determine whether the dualization used in our solution can be applied to the $2 D$ total visibility-index computation to achieve a subquadratic solution on twodimensional terrains. Another interesting avenue for future research is to see if our solution can be applied for faster approximate solutions to the $2 D$ total visibility-index problem by computing total visibility-index on a number of $1 \mathrm{D}$ slices of the $2 \mathrm{D}$ terrain and then using interpolation to approximate visibility indices to all points in the 2D terrain. Finally, this work indicates that persistent data structures can be used as an alternative to plane sweep approaches to achieve performance gains in practice. Furthermore, persistent data structures are easily parallelizable, further improving performance. A practical direction for future research is to apply these persistent structures to improve the performance of the many existing algorithms that use plane sweep approaches.

\section{ACKNOWLEDGMENTS}

This work is supported by the National Science Foundation under Grant No. 1533823.

\section{REFERENCES}

[1] ArcGIS. 2016. Retrieved from http://www.esri.com/software/arcgis.

[2] .GRASS (Geographic Resources Analysis Support System). 2016. Retrieved from https://grass.osgeo.org.

[3] M. J. Atallah, M. T. Goodrich, and S. R. Kosaraju. 1994. Parallel algorithms for evaluating sequences of setmanipulation operations. F. ACM 41, 6 (1994), 1049-1088.

[4] B. Ben-Moshe, O. Hall-Holt, M. J. Katz, and J. S. B. Mitchell. 2004. Computing the visibility graph of points within a polygon. In Proceedings of the 20th Annual Symposium on Computational Geometry (SCG'04). 27-35.

[5] F. Chao, Y. Chongjun, C. Zhuo, Y. Xiaojing, and G. Hantao. 2011. Parallel algorithm for viewshed analysis on a modern GPU. Int. F. Digital Earth 4, 6 (2011), 471-486.

[6] B. Chazelle and H. Edelsbrunner. 1987. Linear space data structures for two types of range search. Discr. Comput. Geom. 2, 2 (Jun. 1987), 113-126.

[7] M. de Berg, O. Cheong, M. van Kreveld, and M. Overmars. 2008. Computational Geometry: Algorithms and Applications (3rd ed.). Springer-Verlag.

[8] J. R. Driscoll, N. Sarnak, D. D. Sleator, and R. E. Tarjan. 1986. Making data structures persistent. In Proceedings of the 18th Annual ACM Symposium on Theory of Computing (STOC'86). 109-121.

[9] C. Ferreira, M. V. Andrade, S. V. Magalhaes, W. R. Franklin, and G. C. Pena. 2013. A parallel sweep line algorithm for visibility computation. In Proceedings of the Symposium on GeoInformatics (GeoInfo'13). 85-96.

[10] C. R. Ferreira, S. V. G. Magalhaes, M. V. A. Andrade, W. R. Franklin, and A. M. Pompermayer. 2012. More efficient terrain viewshed computation on massive datasets using external memory. In Proceedings of the 20th International Conference on Advances in Geographic Information System. 494-497.

[11] J. Fishman, H. Haverkort, and L. Toma. 2009. Improved visibility computation on massive grid terrains. In Proceediings of the 17th ACM SIGSPATIAL International Conference on Advances in Geographic Information Systems. 121-130.

[12] L. De Floriani and P. Magillo. 2003. Algorithms for visibility computation on terrains: A survey. Environ. Plan. B: Plan. Des. 30, 5 (2003), 709-728.

[13] W. R. Franklin and C. K. Ray. 1994. Higher isn't necessarily better: Visibility algorithms and experiments. In Proceedings of Advances in GIS Research: 6th International Symposium on Spatial Data Handling. 751-770.

[14] S. Friedrichs, M. Hemmer, J. King, and C. Schmidt. 2016. The continuous 1.5D terrain guarding problem: Descretization, optimal solutions, and PTAS. f. Comput. Geom. 7, 1 (2016), 256-284. 
[15] A. Haas and M. Hemmer. 2015. Efficient algorithms and implementations for visibility in 1.5D terrains. In Proceedings of the 31st European Workshop on Computational Geometry. 216-219.

[16] T. Hagerup. 1998. Sorting and searching on the word RAM. In Proceedinsg of the 15th Symposium on Theoretical Aspects of Computer Science. 366-398.

[17] H. Haverkort, L. Toma, and Y. Zhuang. 2009. Computing visibility on terrains in external memory. F. Exp. Algor. 13 (2009), 5:1.5-5:1.23.

[18] F. Hurtado, M. Löffler, I. Matos, V. Sacristán, M. Saumell, R. Silveira, and F. Staals. 2014. Terrain visibility with multiple viewpoints. Int. f. Comput. Geom. App. 24, 04 (Dec. 2014)

[19] J. JáJá. 1992. An Introduction to Parallel Algorithms (1st ed.). Addison Wesley.

[20] D. B. Kidner, P. J. Rallings, and J. A. Ware. 1996. Parallel processing for terrain analysis in GIS: Visibility as a case study. GeoInformatica 1, 2 (1996), 183-207.

[21] M. Llobera, D. Wheatley, J. Steele, S. Cox, and O. Parchment. 2004. Calculating the inherent visual structure of a landscape (inherent viewshed) using high-throughput computing. In Proceedings of Beyond the Artifact: Digital Interpretation of the Past: The 32nd Computer Applications and Quantitative Methods in Archaeology Conference (CAA'04). 146-151.

[22] M. Löffler, M. Saumell, and R. I. Silveira. 2014. A faster algorithm to compute the visibility map of a 1.5 D terrain. In Proceedingsf of the 30th European Workshop on Computational Geometry.

[23] L. Palazzi and J. Snoeyink. 1994. Counting and reporting red/blue segment intersections. CVGIP 56, 4 (1994), 304-310.

[24] S. Tabik, A. Cervilla, E. Zapata, and L. Romero. 2014. Efficient data structure and highly scalable algorithm for totalviewshed computation. IEEE 7. Select. Top. Appl. Earth Observ. Remote Sens. 8, 1 (2014), 1-7.

[25] The CGAL Project. 2017. CGAL User and Reference Manual (4.11 ed.). CGAL Editorial Board. Retrieved from http:// doc.cgal.org/4.11/Manual/packages.html

[26] M. van Kreveld. 1996. Variations on sweep algorithms: Efficient computation of extended viewsheds and class intervals. In Proceedings of the 7th International Symposium on Spatial Data Handling. 13-15.

[27] D. Wheatley. 1995. Cumulative Viewshed Analysis: A GIS-based Method for Investigating Intervisibility and Its Archaeological Application. Routlege, London.

[28] Y. Zhao, A. Padmanabhan, and S. Wang. 2013. A parallel computing approach to viewshed analysis of large terrain data using graphics processing units. Int. f. Geogr. Inf. Sci. 27, 2 (2013), 363-384.

[29] R. J. Zomer, D. A. Bossio, A. Trabucco, L. Yuanjie, D. C. Gupta, and V. P. Singh. 2007. Trees and Water: Smallholder Agroforestry on Irrigated Lands in Northern India. Technical Report 122. International Water Management Institute, Colombo, Sri Lanka. 45 pages.

[30] R. J. Zomer, A. Trabucco, D. A. Bossio, O. van Straaten, and L. V. Verchot. 2008. Climate change mitigation: A spatial analysis of global land sustainability for clean development mechanism afforestation and reforestation. Agric. Ecosyst. Environ. 126 (2008), 67-80.

Received May 2017; revised February 2018; accepted April 2018 